\title{
Why fast magnetic reconnection is so prevalent
}

\author{
Allen H. Boozer $\dagger$ \\ Columbia University, New York, NY 10027, USA \\ (Received 9 May 2017; revised 31 December 2017; accepted 4 January 2018)
}

Evolving magnetic fields are shown to generically reach a state of fast magnetic reconnection in which magnetic field line connections change and magnetic energy is released at an Alfvénic rate. This occurs even in plasmas with zero resistivity; only the finiteness of the mass of the lightest charged particle, an electron, is required. The speed and prevalence of Alfvénic or fast magnetic reconnection imply that its cause must be contained within the ideal evolution equation for magnetic fields, $\partial \boldsymbol{B} / \partial t=\nabla \times(\boldsymbol{u} \times \boldsymbol{B})$, where $\boldsymbol{u}(\boldsymbol{x}, t)$ is the velocity of the magnetic field lines. For a generic $\boldsymbol{u}(\boldsymbol{x}, t)$, neighbouring magnetic field lines develop a separation that increases exponentially, as $e^{\sigma(\ell, t)}$ with $\ell$ the distance along a line. This exponentially enhances the sensitivity of the evolution to non-ideal effects. An analogous effect, the importance of stirring to produce a large-scale flow and enhance mixing, has been recognized by cooks through many millennia, but the importance of the large-scale flow $\boldsymbol{u}$ to reconnection is customarily ignored. In part this is due to the sixty-year focus of recognition theory on two-coordinate models, which eliminate the exponential enhancement that is generic with three coordinates. A simple three-coordinate model is developed, which could be used to address many unanswered questions.

Key words: astrophysical plasmas, fusion plasma

\section{Introduction}

An evolving magnetic field that is embedded in a highly conducting plasma generically undergoes fast magnetic reconnections, which means with a reconnection speed determined not by resistive but by Alfvénic effects (Comisso \& Bhattacharjee 2016; Loureiro \& Uzdensky 2016; Zweibel \& Yamada 2016). Reconnection at a speed of a tenth of the Alfvén speed is common in both observations and experiments (Comisso \& Bhattacharjee 2016). Since fast reconnection is both prevalent and fast compared to resistive time scales, its cause must be within the ideal evolution of magnetic fields, and this will be shown to be true. Fast magnetic reconnection is a quasi-ideal process with a conservation law, helicity conservation, which does not hold on a resistive time scale (Boozer 2017).

The properties of three-dimensional reconnection presented here are direct consequences of Maxwell's equations and standard mathematics. Nevertheless, their unconventionality makes acceptance difficult. For sixty years, reconnection has been viewed as essentially a two-dimensional process even in three-dimensional space (Comisso \& Bhattacharjee 2016; Loureiro \& Uzdensky 2016; Zweibel \& Yamada

$\dagger$ Email address for correspondence: ahb17@columbia.edu 
2016). The prevalence of fast magnetic reconnection is recognized, but the reason has not been a focus of research. Two-coordinate models can explain how reconnection can be fast but not why fast reconnection is so prevalent. The easiest way to avoid an unconventional conclusion is to not take the time to understand the derivation. To minimize that reason for avoidance, the most important derivations are placed in $\S \S 1$ and 2 and are self-contained.

Two-coordinate systems do not naturally evolve from smooth to rapidly reconnecting states. Fast reconnection is possible in two-coordinate systems in which the initial state is singular - a state that could not naturally arise. A Harris (1962) sheet is a common example of such a non-realizable state (Boozer 2014). Another is magnetic flux ropes in which the magnetic field is strong throughout the rope but zero outside, which violates the requirement that the magnetic field not only be a continuous but also a differentiable function of position to satisfy Maxwell's equations.

Three-coordinate models explain why magnetic reconnection is both fast and generic. Nature is three-dimensional, so two-coordinate models can only be an approximation. Three-dimensional theory is required to understand the range of validity of two-coordinate approximations.

Reconnection in this paper is defined as the changing of the connections of magnetic field lines. Consequently, there is no reconnection when a magnetic field evolves as if it were embedded in a perfectly conducting fluid moving with a velocity $\boldsymbol{u}$. In this definition, the motion of magnetic field lines relative to a plasma in which they are embedded need not imply reconnection.

Magnetic reconnection is sometimes defined as the breaking of the ideal constraint between plasma and magnetic field line motions. For example, Eyink (2015) defined magnetic reconnection by the breaking 'of magnetic connections between plasma elements'. With this definition, fluid turbulence can enhance reconnection (Eyink, Lazarian \& Vishniac 2011) in a way that is not possible within the definition used in this paper. One might note that the Eyink definition implies that a time-independent magnetic field in a stellarator is reconnecting as the plasma diffuses across it.

Boozer (2004) noted that the evolution of a magnetic field that is embedded in a perfectly conducting fluid obeys two distinct conservation laws: (i) the ideal evolution of $\boldsymbol{B}$, and (ii) the tying of the magnetic field lines to the fluid. Only the breaking of the first of these two conservation laws is relevant to reconnection as defined in this paper, and this conservation law holds far more accurately in tokamak experiments than the second, appendix A. As will be seen, plasma turbulence can enhance the breaking of magnetic connections, but as explained in $\$ 2.1$, the effect is intrinsically weaker than non-symmetric effects with a long spatial scale across the magnetic field lines.

The evolution of a magnetic field is determined by the electric field, $\partial \boldsymbol{B} / \partial t=-\nabla \times$ $\boldsymbol{E}$. The magnetic field evolution is by definition ideal wherever the electric field can be written in the form

$$
\boldsymbol{E}+\boldsymbol{u} \times \boldsymbol{B}=-\nabla \Phi,
$$

with $\boldsymbol{u}(\boldsymbol{x}, t)$, the magnetic field line velocity, and $\Phi(\boldsymbol{x}, t)$ well-behaved functions of position. Newcomb (1958) gave a proof that $\boldsymbol{u}$ is the velocity of the magnetic field lines; Boozer (2010) gave a much simpler proof. The component of $\boldsymbol{u}$ along $\boldsymbol{B}$ has no role in reconnection theory and will be assumed to be zero to simplify the discussion.

Equation (1.1) implies an anti-reconnection theorem, which was first recognized by Newcomb (1958). The proof of the theorem is obvious - any vector $\boldsymbol{E}(\boldsymbol{x}, t)$, can be represented locally in the form of (1.1). Let $\Phi$ be a solution to $\boldsymbol{B} \cdot \nabla \Phi=-\boldsymbol{B} \cdot \boldsymbol{E}$, or equivalently $\mathrm{d} \Phi / \mathrm{d} \ell=-E_{\|}$, where $\mathrm{d} \ell$ is the differential distance along a magnetic 
field line. Define $\boldsymbol{u}$ so $\boldsymbol{u}=(\boldsymbol{E}+\nabla \Phi) \times \boldsymbol{B} / B^{2}$. This anti-reconnection theorem led Greene (1993) to the conclusion that (1.1) 'is purely geometrical, and has no physical content'. The magnetic field line velocity $\boldsymbol{u}(\boldsymbol{x}, t)$ is subtle but does have important physical implications. The subtlety is in the freedom of choice of the variation of $\Phi$ across the magnetic field lines at $\ell=0$ when integrating $\mathrm{d} \Phi / \mathrm{d} \ell=-E_{\|}$. Magnetic field lines are given by a Hamiltonian, and the freedom of canonical transformations of the Hamiltonian is equivalent to the freedom in $\Phi$ at $\ell=0$ (Boozer 2004).

The separation of the velocity $\boldsymbol{u}$ of the magnetic field lines from the velocity $\boldsymbol{v}$ of the plasma and the anti-reconnection theorem remain important in relativistic theory, appendix B.

Equation (1.1) implies that magnetic reconnection requires $\Phi$ or $\boldsymbol{u}$ be ill behaved. As noted by Greene (1993), this is true at nulls of $\boldsymbol{B}$. Nevertheless, it is difficult to understand how magnetic field nulls can explain why natural magnetic fields generically evolve into states in which fast magnetic reconnection occurs. As shown in appendix C.2, field nulls are (i) generically spatially rare, and (ii) a magnetic field line that intercepts one null does not generally intercept other nearby nulls. A field line intercepting two nulls is a more singular situation than a line intercepting only a single null. An ideal evolution without a null cannot produce a null, $\S 2.3$. As shown by Boozer (2010), in a generic evolution nulls can only be produced in pairs and only at discrete space-time points. Field nulls are clearly not required for a fast reconnection because fast magnetic reconnections are observed in tokamak plasmas, which have no magnetic nulls.

Magnetic reconnection can also occur when a well-behaved solution to $\mathrm{d} \Phi / \mathrm{d} \ell=$ $-E_{\|}$does not exist because of boundary conditions on the electric field. This can occur when the field lines intercept perfect conductors, or essentially equivalently when $\Phi$ must obey periodicity conditions as in toroidal plasmas. Such boundary or periodicity conditions are trivially satisfied when $E_{\|}=0$, so a non-zero $E_{\|}$is a necessary condition for reconnection.

A remarkable feature of three-coordinate models is that as a magnetic field undergoes an ideal evolution, which means (1.1) is satisfied exactly, the sensitivity of field line connections to $E_{\|}$increases exponentially, as $e^{\sigma}$. This is due to the exponentially increasing separation of neighbouring field lines, as $e^{\sigma(\ell, t)}$, with distance $\ell$ along a line. An arbitrarily small $E_{\|}$can produce an Alfvén speed reconnection on the overall scale of the system. This has an analogy in the mixing of fluids. Exponential separation of fluid elements in time explains the effectiveness of stirring for the intermixing of fluids, such as the stirring of cream in coffee. As explained in $\$ 2.1$, exponentiation in fluid mixing requires only two spatial coordinates but in magnetic reconnection requires three spatial coordinates.

The time required for an initial magnetic field to arrive at adequate exponential sensitivity defines the trigger time for a fast reconnection. Once reconnection occurs, an Alfvénic relaxation is required before the system can re-establish a quasi-static force balance no matter how slow or weak the external drive for the magnetic field evolution may be, $\S 2.5$. The anti-reconnection theorem, which follows from (1.1), implies the specific location of the reconnection event does not generally have a unique definition. The analogy to the trigger time in the mixing of cream into coffee is the time required for the streamers of cream to become sufficiently thin that molecular diffusion can intermix the cream and coffee on a molecular level.

No matter how slow the external forcing of an ideally evolving magnetic field may be, energy can be accumulated in the magnetic field. Much of that energy is released on a Alfvénic time scale when the exponentiation becomes sufficiently 
great to trigger a fast magnetic reconnection. As discussed in Boozer (2017), a fast magnetic reconnection conserves magnetic helicity, which implies the full energy stored in the field cannot be released - only that associated with $\left|\nabla\left(j_{\|} / B\right)\right|$ (Woltjer 1958). Equation (3.34) shows that when magnetic field lines with different $j_{\|} / B$ are connected, a Lorentz force $\boldsymbol{f}=\boldsymbol{j} \times \boldsymbol{B}$ is exerted on the plasma. When dissipation is small, this force is balanced by the plasma inertia, $\rho \mathrm{d} v / \mathrm{d} t$ and the energy released by the relaxation of $\left|\nabla\left(j_{||} / B\right)\right|$ goes into Alfvén waves. The damping of these waves transfers the energy to the plasma. This process is discussed in $\S \S 3$ and 4 .

In three dimensions, unlike in two, the magnetic energy density $B^{2} / 2 \mu_{0}$ need not become large to obtain an arbitrarily large exponential increase when neighbouring magnetic field lines separate exponentially. Consequently, there is no natural tendency for the back reaction of the system to suppress this exponentiation. This is unlike the situation discussed by Cattaneo \& Hughes (1996) in connection with the theory of dynamos. This is like the stirring of cream into coffee in which there is no back reaction that impedes the formation of narrow streamers of cream.

Section 3.8 shows the computational difficulty of studying reconnection in three spatial dimensions increases as $e^{5 \sigma}$. This appears to limit direct simulations to $\sigma_{\max } \approx$ 10 , which is consistent with a $\sigma \approx 8$ required to understand fast reconnection in fusion plasmas but much smaller than $\sigma \approx 20$ required to understand reconnection in the solar corona. Simulations using modest values of $\sigma$ must be sufficiently well understood to devise extrapolations or reliable approximations.

The computational difficulty of studying fast reconnection is sufficiently great that simplified models are required. Because exponentiation is an effect of critical importance and dominant, a simplified model, such as the one given in $\S 3$, can answer many of the most important questions, $\S 4$.

Models related to the model of $\S 3$ could be used to directly study reconnection in the solar corona, $\S \mathrm{C} .3$, although the required number of exponentiations $\sigma \approx 20$ is too large for complete realism in a simulation. Such models would predict the energy of electrons in the corona and the height of the transition region to the corona. The short scale height of the plasma density below the transition region, $\sim 100 \mathrm{~km}$, implies that there are too few electrons to carry the current required by a quasi-ideal magnetic evolution even at a height far below the scale of magnetic phenomena on the Sun, $\sim 10^{4} \mathrm{~km}$. Without a corona, the electron density would drop over $10^{43}$ times over a radial scale of $10^{4} \mathrm{~km}$. Electrons runaway to whatever energy is required to carry the current, which gives a corona. This occurs where the mean free path of electrons, $\lambda_{\mathrm{mfp}}$, times parallel electric field becomes comparable to the local electron temperature, $\lambda_{\text {mfp }} e E_{\|} \sim T_{e}$, where $E_{\|}=\eta j_{\|}$. The requirement for a corona was shown in Boozer (1999) and discussed on page 1092 of Boozer (2004).

The fast-reconnection mechanism explained in this paper is a clear implication of Maxwell's equations. A different mechanism could break the ideal evolution before the exponential sensitivity to $E_{\|}$does. Having a definite three-coordinate model, such as the one presented in $\S 3$, allows one to explore under what conditions the standard two-coordinate models are an adequate approximation.

\section{Ideal magnetic evolution}

\subsection{Exponentiation and reconnection}

Magnetic field lines are defined by $\mathrm{d} \boldsymbol{x} / \mathrm{d} \ell=\hat{b}(\boldsymbol{x})$, where $\hat{b} \equiv \boldsymbol{B} /|\boldsymbol{B}|$ is the unit vector and $\ell$ is the distance along the magnetic field. The position of a second field line is $\boldsymbol{x}+\boldsymbol{\delta}$. By definition a neighbouring line satisfies $|\boldsymbol{\delta}| \rightarrow 0$. For a neighbouring line, 
$\mathrm{d} \boldsymbol{\delta} / \mathrm{d} \ell=(\boldsymbol{\delta} \cdot \nabla) \hat{b}$. This linear equation for $\boldsymbol{\delta}$ holds as long as $|\boldsymbol{\delta}|<<a_{c}$, where $a_{c}$ is the characteristic spatial scale within which the linear term in a Taylor expansion of $\hat{b}$ dominates over the higher-order terms.

A plasma cannot respond to maintain magnetic connectivity on a smaller spatial scale than $c / \omega_{\text {pe }}$ set by the inertia of the lightest charged particle, an electron. A nonideal parallel electric field is required $E_{\|}=\left(m_{e} / e^{2}\right) \mathrm{d}\left(j_{\|} / n_{e}\right) / \mathrm{d} t$ to accelerate electrons of number density $n_{e}$ to carry the required current $j_{\|}$along the magnetic field lines; $\left(c / \omega_{\mathrm{pe}}\right)^{2} \equiv m_{e} /\left(\mu_{0} n_{e} e^{2}\right)$. In a force-free magnetic field, $\nabla \times \boldsymbol{B}=\mu_{0} \boldsymbol{j}_{\|}$, so when electron inertia is the only correction to the ideal evolution,

$$
\frac{\partial}{\partial t}\left(\boldsymbol{B}-\left(\frac{c}{\omega_{\mathrm{pe}}}\right)^{2} \nabla^{2} \boldsymbol{B}\right)=\nabla \times(\boldsymbol{u} \times \boldsymbol{B}) .
$$

The $\nabla^{2} \boldsymbol{B}$ term smears out the location of the magnetic field over a scale $c / \omega_{\mathrm{pe}}$. This smearing effect is seen when the right-hand side of (2.1) is replaced by resistive diffusion $\left(\eta / \mu_{0}\right) \nabla^{2} \boldsymbol{B}$, and the equation solved with $\boldsymbol{B}=B(t) \sin (k x) \hat{z}$. The solution is $B(t)=B_{0} e^{-v t}$ with $v=\left(\eta / \mu_{0}\right) k^{2} /\left(1+\left(c / \omega_{\mathrm{pe}}\right)^{2} k^{2}\right)$. Magnetic field lines that are within a distance of $c / \omega_{\mathrm{pe}}$ of each other at one location along their trajectories can reconnect freely even if they are separated by a distance up to $a_{c}$ at some other trajectory location.

When the initial magnetic field is a constant, $B_{0} \hat{z}_{0}$, a flux tube of circular cross-section contains flux $\pi B_{0} \delta_{0}^{2}$, which remains fixed during an ideal evolution. In the limit $\delta_{0} \rightarrow 0$, the tube evolves into an elliptical cross-section with major and minor radii $\delta_{\text {maj }}$ and $\delta_{\text {min }}$ but with the same flux, $\pi B \delta_{\text {maj }} \delta_{\text {min }}=\pi B_{0} \delta_{0}^{2}$. The major radius grows as $\delta_{\text {maj }}=\delta_{0} e^{\sigma(\ell, t)}$ with time and distance along the tube, where $\sigma$ is a Lyapunov exponent associated with the flow $\boldsymbol{u}$. A precise definition of $\sigma$ is given in $\S 2.3$. The minor radius must shrink as $\delta_{\min }=\left(B_{0} / B\right) \delta_{0} e^{-\sigma(\ell, t)}$. When the initial radius $\delta_{0}$ is made finite, the major radius can become larger than the characteristic spatial scale $a_{c}$. When $\delta_{\text {maj }}$ becomes greater than $a_{c}$, the flux tube deforms into an extremely complicated shape and the maximum distance between points in a cross-section of the flux tube increases only as $\ell$ to a power, which is the case considered by Rechester $\&$ Rosenbluth (1978). For this reason, short wavelength plasma turbulence intrinsically produces a less dramatic increase in the reconnection rate than the exponential increase caused by variations in the field of longer wavelength. For spatial scales $a<a_{c}$, fast magnetic reconnection can occur over the scale $a$ when the change in the exponentiation along a magnetic field line $\delta \sigma$ satisfies $\delta \sigma \sim \ln \left(a /\left(c / \omega_{\mathrm{pe}}\right)\right)$.

The transition in the form of the separation of magnetic field lines from exponential when $\delta_{\text {maj }}<a_{c}$ to a power-law, $\ell^{\alpha}$, is complicated. Analogous issues arise in the mixing of fluids and its practical applications - even stirring cream in coffee. Two articles in the Reviews of Modern Physics help clarify the issues. The limit corresponding to $\delta_{\text {maj }}<a_{c}$ is the advective or stirring limit for fluids and the opposite limit is the mixing or turbulent limit. Both reviews consider the two limits. The earlier review of Falkovich, Gawdcezki \& Vergassola (2001) emphasized the turbulent limit and a recent review by Aref et al. (2017) emphasized the advective limit. Separations between fluid elements typically increase exponentially with time in the advective limit but as time to a power in turbulent limit. Different powers of time arise depending on assumptions. Numerical studies of the field line motion in models of turbulent magnetic fields were given in Zimbardo et al. (1995). The separation between neighbouring magnetic field lines were observed to increase as a power law, $\ell^{\alpha}$, with the power depending on the assumptions of the model. 
An important distinction exists between mixing in a two-coordinate model of an incompressible flow, such as $\boldsymbol{v}=\nabla \phi(x, y, t) \times \hat{z}$, and reconnection in a two-coordinate model of the magnetic field, such as $\boldsymbol{B}=B_{g}(\hat{z}+\nabla H(x, y, t) \times \hat{z})$, where $B_{g}$ is a strong and constant guide field. Both streamlines and magnetic field lines obey Hamilton's equations. For streamlines, $\mathrm{d} x / \mathrm{d} t=\partial \phi / \partial y$ and $\mathrm{d} y / \mathrm{d} t=-\partial \phi / \partial x$. For magnetic field lines, $\mathrm{d} x / \mathrm{d} z=\partial H / \partial y$ and $\mathrm{d} y / \mathrm{d} z=-\partial H / \partial x$, so $\mathrm{d} H / \mathrm{d} z=$ $(\partial H / \partial x)(\mathrm{d} x / \mathrm{d} z)+(\partial H / \partial y)(\mathrm{d} y / \mathrm{d} z)=0$. With only two coordinates, a magnetic field line must follow a constant- $H$ contour. No such constraint applies to streamlines in a time-dependent flow. Although a rapid mixing of fluids can occur with time-dependent stirring in a two-coordinate model, a three-coordinate model is required for the analogous effect to enhance magnetic reconnection.

In the solar corona, $c / \omega_{\mathrm{pe}} \sim 10 \mathrm{~cm}$, and the radius of the Sun is approximately ten orders of magnitude greater, $R_{\odot} \approx 7 \times 10^{5} \mathrm{~km}$, so an exponentiation $\sigma \sim 23$ could be responsible for all observed reconnection events. An even larger $\sigma$ may arise in astrophysical reconnection. A much smaller $\sigma$ is required to explain fast reconnections in fusion experiments. The minor radius of ITER is $a=2.0 \mathrm{~m}$, and the standard operating density is $10^{20}$ electrons $\mathrm{m}^{-3}$, which makes $a /\left(c / \omega_{\mathrm{pe}}\right)=e^{8.2}$.

\subsection{Ideal form for $\boldsymbol{B}(\boldsymbol{x}, t)$}

A magnetic field $\boldsymbol{B}(\boldsymbol{x}, t)$ maintains a special form, equation (2.8), when it is undergoing an arbitrary ideal evolution from an initial state $\boldsymbol{B}_{0}\left(\boldsymbol{x}_{0}\right)$. This form is based on the transformation to Lagrangian coordinates, $\boldsymbol{x}\left(\boldsymbol{x}_{0}, t\right)$,

$$
\frac{\mathrm{d} \boldsymbol{x}}{\mathrm{d} t} \equiv \boldsymbol{u}(\boldsymbol{x}, t)
$$

with the initial condition $\boldsymbol{x}\left(\boldsymbol{x}_{0}, t_{0}\right)=\boldsymbol{x}_{0}$ and with $\boldsymbol{u}(\boldsymbol{x}, t)$ the velocity in (1.1). When $f(\boldsymbol{x}, t)$ is an arbitrary function, $\mathrm{d} f / \mathrm{d} t \equiv \partial f / \partial t+\boldsymbol{u} \cdot \nabla f$. Since $\boldsymbol{x}_{0}$ is not changed by the flow, $\mathrm{d} f / \mathrm{d} t=(\partial f / \partial t)_{x_{0}}$.

The Jacobian matrix of the coordinate transformation $\boldsymbol{x}\left(\boldsymbol{x}_{0}, t\right)$ is

$$
\boldsymbol{J} \equiv \frac{\partial \boldsymbol{x}}{\partial \boldsymbol{x}_{0}} \equiv\left(\begin{array}{ccc}
\frac{\partial x}{\partial x_{0}} & \frac{\partial x}{\partial y_{0}} & \frac{\partial x}{\partial z_{0}} \\
\frac{\partial y}{\partial x_{0}} & \frac{\partial y}{\partial y_{0}} & \frac{\partial y}{\partial z_{0}} \\
\frac{\partial z}{\partial x_{0}} & \frac{\partial z}{\partial y_{0}} & \frac{\partial z}{\partial z_{0}}
\end{array}\right)
$$

The Jacobian $\mathcal{J}$ is the determinant of the Jacobian matrix, $\mathcal{J} \equiv|\boldsymbol{J}|$.

The time derivative of the Jacobian can be derived using an arbitrary volume moving with the flow, $V \equiv \int \mathcal{J} \mathrm{d}^{3} x_{0}$. This volume changes as $\mathrm{d} V / \mathrm{d} t=\oint \boldsymbol{u} \cdot \mathrm{d} \boldsymbol{a}=$ $\int \boldsymbol{\nabla} \cdot \boldsymbol{u} \mathrm{d}^{3} x=\int \boldsymbol{\nabla} \cdot \boldsymbol{u} \mathcal{J} \mathrm{d}^{3} x_{0}$. Since $\mathrm{d} V / \mathrm{d} t=\int(\mathrm{d} \mathcal{J} / \mathrm{d} t) \mathrm{d}^{3} x_{0}$ holds for an arbitrary volume,

$$
\frac{\mathrm{d} \mathcal{J}}{\mathrm{d} t}=\mathcal{J} \nabla \cdot \boldsymbol{u}
$$

In an ideal evolution, $\partial \boldsymbol{B} / \partial t=\nabla \times(\boldsymbol{u} \times \boldsymbol{B})=-\boldsymbol{B} \nabla \cdot \boldsymbol{u}+\boldsymbol{B} \cdot \nabla \boldsymbol{u}-\boldsymbol{u} \cdot \nabla \boldsymbol{B}$, so

$$
\frac{\mathrm{d}(\mathcal{J} \boldsymbol{B})}{\mathrm{d} t}=(\mathcal{J} \boldsymbol{B}) \cdot \nabla \boldsymbol{u}
$$


The general expression for $\boldsymbol{B} \cdot \nabla f$ is apparent from its two-coordinate form

$$
\begin{aligned}
\boldsymbol{B} \cdot \nabla f & =B_{x}\left(\frac{\partial x_{0}}{\partial x} \frac{\partial}{\partial x_{0}}+\frac{\partial y_{0}}{\partial x} \frac{\partial}{\partial y_{0}}\right) f B_{y}\left(\frac{\partial x_{0}}{\partial y} \frac{\partial}{\partial x_{0}}+\frac{\partial y_{0}}{\partial y} \frac{\partial}{\partial y_{0}}\right) f \\
& =\frac{\partial f}{\partial \boldsymbol{x}_{0}} \cdot \frac{\partial \boldsymbol{x}_{0}}{\partial \boldsymbol{x}} \cdot \boldsymbol{B}
\end{aligned}
$$

as is $\boldsymbol{J}^{-1}=\partial \boldsymbol{x}_{0} / \partial \boldsymbol{x}$. Since $\boldsymbol{u} \equiv \partial \boldsymbol{x}\left(\boldsymbol{x}_{0}, t\right) / \partial t$, the derivative $\left(\partial \boldsymbol{u} / \partial \boldsymbol{x}_{0}\right)_{t}=\mathrm{d} \boldsymbol{J} / \mathrm{d} t$. Therefore, equation (2.5) is equivalent to

$$
\frac{\mathrm{d}(\mathcal{J} \boldsymbol{B})}{\mathrm{d} t}=\frac{\mathrm{d} \boldsymbol{J}}{\mathrm{d} t} \cdot \boldsymbol{J}^{-1} \cdot(\mathcal{J} \boldsymbol{B})
$$

which is solved by

$$
\boldsymbol{B}(\boldsymbol{x}, t)=\frac{\boldsymbol{J}}{\mathcal{J}} \cdot \boldsymbol{B}_{0}\left(\boldsymbol{x}_{0}\right),
$$

where $\boldsymbol{B}_{0}\left(\boldsymbol{x}_{0}\right)$ is the initial, $t=t_{0}$, magnetic field. Equation (2.8) is given in the review of reconnection by Zweibel \& Yamada (2016). The long history of this equation was discussed by Stern (1966). Zweibel (1998) used (2.8) to study magnetic reconnection when there is a stagnation point in the plasma flow. Her analysis is related to that given in $\S 2.3$ for the effect on reconnection of a generic magnetic field line velocity.

\subsection{Ideal $\boldsymbol{B}(\boldsymbol{x}, t)$ and exponentiation}

The Jacobian matrix can be written using a singular value decomposition (SVD), as can any three-by-three matrix with real coefficients,

$$
\boldsymbol{J}=\boldsymbol{U} \cdot\left(\begin{array}{ccc}
e^{\sigma_{1}} & 0 & 0 \\
0 & e^{\sigma_{2}} & 0 \\
0 & 0 & e^{\sigma_{3}}
\end{array}\right) \cdot \boldsymbol{V}^{\dagger}
$$

where $\boldsymbol{U}$ and $\boldsymbol{V}$ are orthogonal matrices, $\boldsymbol{U}^{\dagger} \cdot \boldsymbol{U}=\mathbf{1}$. Orthogonal matrices give rotations. This is obvious for a two-by-two orthogonal matrix, which has the general form

$$
\boldsymbol{U}=\left(\begin{array}{cc}
\cos \alpha & \sin \alpha \\
-\sin \alpha & \cos \alpha
\end{array}\right)
$$

when $\boldsymbol{U}$ goes to the identity matrix as the rotation angle $\alpha$ goes to zero. In an SVD analysis, the coefficients $e^{\sigma_{1}}, e^{\sigma_{2}}$ and $e^{\sigma_{3}}$ are called singular values and are positive real numbers. In the theory of dynamical systems, the real numbers $\sigma_{1-3}$ are known as Lyapunov exponents, or more precisely as finite-time Lyapunov exponents.

The dot product of the magnetic field with itself implies

$$
\left(\frac{\mathcal{J} B}{B_{0}}\right)^{2}=\hat{b}_{0}^{\dagger} \cdot \boldsymbol{V} \cdot\left(\begin{array}{ccc}
e^{2 \sigma_{1}} & 0 & 0 \\
0 & e^{2 \sigma_{2}} & 0 \\
0 & 0 & e^{2 \sigma_{3}}
\end{array}\right) \cdot \boldsymbol{V}^{\dagger} \cdot \hat{b}_{0},
$$

where $\hat{b}_{0}=\boldsymbol{B}_{0} / B_{0}$ is the unit vector along the initial magnetic field. 
The component of $\boldsymbol{u}$ that is parallel to the magnetic field does not appear in (1.1) for an ideal evolution and is taken to be zero, which simplifies the analysis, especially in relativistic theory. With $\boldsymbol{u} \cdot \boldsymbol{B}=0$, magnetic flux conservation during the evolution then implies $\mathcal{J} B=B_{0}$.

Define the transformed unit vector $\hat{b}_{t} \equiv \boldsymbol{V}^{\dagger} \cdot \hat{b}_{0}$, then

$$
\left(\frac{\mathcal{J} B}{B_{0}}\right)^{2}=\hat{b}_{t}^{\dagger} \cdot\left(\begin{array}{ccc}
e^{2 \sigma_{1}} & 0 & 0 \\
0 & e^{2 \sigma_{2}} & 0 \\
0 & 0 & e^{2 \sigma_{3}}
\end{array}\right) \cdot \hat{b}_{t}=1 .
$$

One of the singular values, which will be taken to be $e^{\sigma_{3}}$, must be unity, and $\hat{b}_{t}$ is the associated eigenvector. Consequently,

$$
\begin{aligned}
& \boldsymbol{J}=\boldsymbol{U} \cdot\left(\begin{array}{ccc}
e^{\sigma_{1}} & 0 & 0 \\
0 & e^{\sigma_{2}} & 0 \\
0 & 0 & 1
\end{array}\right) \cdot \boldsymbol{V}^{\dagger} \\
& \boldsymbol{B}=\boldsymbol{U} \cdot\left(\begin{array}{ccc}
e^{-\sigma_{2}} & 0 & 0 \\
0 & e^{-\sigma_{1}} & 0 \\
0 & 0 & e^{-\left(\sigma_{1}+\sigma_{2}\right)}
\end{array}\right) \cdot \boldsymbol{V}^{\dagger} \cdot \boldsymbol{B}_{0} ; \\
& B=\frac{B_{0}}{\mathcal{J}}=B_{0} e^{-\left(\sigma_{1}+\sigma_{2}\right)}
\end{aligned}
$$

The magnetic field strength can change in an ideal evolution, but only exponentially, which is not consistent with the formation of a null where none existed before.

\subsection{Ideal evolution of the current density}

The parallel-current density, which is the component that is directly involved in reconnection, generally increases as the magnetic field evolves. The current density $\boldsymbol{j}=\nabla \times(B \hat{b}) / \mu_{0}=\left(\nabla B / \mu_{0}\right) \times \hat{b}+\left(B / \mu_{0}\right) \nabla \times \hat{b}$, so

$$
\begin{aligned}
K & \equiv \frac{\mu_{0} j_{\|}}{B} \\
& =\hat{b} \cdot \nabla \times \hat{b} .
\end{aligned}
$$

The unit vector along the evolved magnetic field is $\hat{b}=\boldsymbol{W} \cdot \hat{b}_{0}$, where $\boldsymbol{W}=\boldsymbol{U} \cdot \boldsymbol{V}^{\dagger}$ is also an orthogonal matrix. If the initial magnetic field is $\boldsymbol{B}_{0}=B_{0} \hat{z}_{0}$ in $\left(x_{0}, y_{0}, z_{0}\right)$ Cartesian coordinates then, $\hat{b}=\sin \alpha \cos \beta \hat{x}_{0}+\sin \alpha \sin \beta \hat{y}_{0}+\cos \alpha \hat{z}_{0}$. It is the gradients of the angles $\alpha$ and $\beta$ across the magnetic field lines that determine the evolution of the parallel-current density in an ideal evolution.

In the model for an ideal magnetic evolution, $\S 3.5, \mathrm{~d} K / \mathrm{d} t=\mathrm{d} \Omega / \mathrm{d} \ell$, where $\mathrm{d} / \mathrm{d} t$ is the time derivative in the frame of the magnetic field lines and $\mathrm{d} / \mathrm{d} \ell$ is the derivative along a magnetic field line. $\Omega$ is the component of the flow vorticity aligned with the magnetic field. The evolution in this model is driven by a flow in a perfectly conducting boundary with $\Omega_{w}^{(s)}(t)$ the flow vorticity at fixed fluid points in the flow of the boundary. When $\Omega_{w}^{(s)}(t)$ evolves slowly compared to the Alfvén transit time from one boundary to the other, $\tau_{A} \equiv L / V_{A}$, then $K(t)=t \bar{\Omega}_{w}^{(s)} / L$, where the timeaveraged vorticity is $\bar{\Omega}_{w}^{(s)} \equiv\left(\int_{0}^{t} \Omega_{w}^{(s)}\left(t^{\prime}\right) \mathrm{d} t^{\prime}\right) / t$. Within this model, it is difficult to see 
how a singular current density can be created. Although $K$ characteristically increases linearly in time, the exponentiation in distance between trajectories $\sigma$ can become large. Boozer (2012) found that the maximum possible $\sigma$ is proportional to $K$. The characteristic increase in the exponentiation $\sigma$ is linear in time in an ideal evolution.

A singular parallel current, called a sheet or delta-function current, can occur in an ideal evolution on the rational surfaces of topologically toroidal plasmas (Boozer \& Pomphrey 2010). These surfaces on which the magnetic field lines close on themselves are spatially isolated. It is difficult to imagine an analogue of a surface of perfectly closed field lines in a naturally occurring magnetic field.

The role of current sheets in fast magnetic reconnection has limitations related to magnetic field nulls. Both are difficult to produce, both are spatially isolated and neither is required in order to obtain reconnection at an Alfvénic speed.

\subsection{Initiation of reconnection}

When a magnetic evolution can be accurately approximated as ideal, both the sensitivity to the breaking of the ideal constraints and the magnitude of the breaking increase exponentially until a fast reconnection occurs. The time required to initiate a fast reconnection is called the trigger time.

Once the fast reconnection starts, the system is taken out of static force balance, and inertial or viscous forces are required. When force balance is maintained by inertia, the relaxation is by Alfvén waves. The relaxation can be complicated since the exponentiation $\sigma$ can change over the entire system during a relaxation, whichever is dominant inertia or viscosity. The relaxation can be studied in a simplified model such as that of $\S 3$.

\section{Simple reconnection model}

\subsection{Model definition}

Features of reconnection that arise in magnetic field structures that depend on all three spatial coordinates can be studied in highly simplified models since many features are generic. A model will be developed based on reduced magnetohydrodynamics (MHD) (Kadomtsev \& Pogutse 1974; Strauss 1976) in which the magnetic field has the form (van Ballegooijen 1985; Ng, Lin \& Bhattacharjee 2012)

$$
\boldsymbol{B}=B_{g}\left(\hat{z}+\nabla_{\perp} H \times \hat{z}\right)
$$

in Cartesian coordinates, where $B_{g}$ is a constant guide field and $\nabla_{\perp}=\hat{x} \partial / \partial x+\hat{y} \partial / \partial y$.

Van Ballegooijen used the magnetic field of (3.1) to study whether current singularities would develop but did not directly study magnetic reconnection.

The model system extends from a wall at $z=0$ to a wall at $z=L$ with $L\left|\nabla_{\perp} H\right|$ remaining finite as $L \rightarrow \infty$. The $z=0$ wall is a rigid perfect conductor, but the $z=L$ wall is a flowing perfect conductor that has a velocity $\boldsymbol{v}_{w}=\nabla_{\perp} \phi_{w} \times \hat{z}$. The guide field $B_{g}$ is too strong to be compressed, so the velocity of the plasma that lies in the region $0<z<L$ can be assumed to have the velocity

$$
v=\nabla_{\perp} \phi \times \hat{z}
$$

where $\phi(x, y, z=L, t)=\phi_{w}(x, y, t)$, which is the streamfunction in the flowing wall. Energy is put into the system by the moving wall and in steady state must be removed by dissipation. 
In addition to $B_{g} H$ being the $\hat{z}$ component of the vector potential, $H(x, y, z, t)$ is the Hamiltonian for the magnetic field lines with $t$ a parameter,

$$
\begin{aligned}
& \frac{\mathrm{d} x}{\mathrm{~d} z}=\frac{\partial H}{\partial y} \\
& \frac{\mathrm{d} y}{\mathrm{~d} z}=-\frac{\partial H}{\partial x} .
\end{aligned}
$$

Magnetic field line trajectories are given by a Hamiltonian of the same type as $H(x, y, z, t)$ in far more general representations of the magnetic field than that of (3.1). The magnetic field in a stellarator or tokamak can always be represented as (Boozer 1983, 2015)

$$
2 \pi \boldsymbol{B}=\nabla \psi_{t} \times \nabla \theta+\nabla \varphi \times \nabla \psi_{p}\left(\psi_{t}, \theta, \varphi, t\right),
$$

where the poloidal flux $\psi_{p}$ is the field line Hamiltonian: $\mathrm{d} \theta / \mathrm{d} \varphi=\partial \psi_{p} / \partial \psi_{t}$ and $\mathrm{d} \psi_{t} / \mathrm{d} \varphi=-\partial \psi_{p} / \partial \theta$. The toroidal magnetic flux is $\psi_{t}$, the poloidal angle is $\theta$ and the toroidal angle is $\varphi$.

The streamfunction $\phi(x, y, z, t)$ is the Hamiltonian that describes the motion of plasma points in a constant $-z$ plane,

$$
\begin{aligned}
& \frac{\mathrm{d} x}{\mathrm{~d} t}=\frac{\partial \phi}{\partial y} \\
& \frac{\mathrm{d} y}{\mathrm{~d} t}=-\frac{\partial \phi}{\partial x} .
\end{aligned}
$$

That is, $z$ is a parameter in the Hamiltonian $\phi(x, y, z, t)$ and not one of the canonical variables.

\subsection{Dimensionality and exponentiation}

The fundamental difference in magnetic reconnection in two- versus three-coordinate systems is contained in the Hamiltonian description of magnetic field line trajectories. At each point in time, the Hamiltonian for magnetic field line trajectories, equations (3.3) and (3.4), depends on the same number of coordinates as the magnetic field. In the literature, a Hamiltonian $H(x, y)$ is said to be a one-degree-of-freedom Hamiltonian. A Hamiltonian $H(x, y, z)$ is said to be a one-and-a-half-degree-offreedom Hamiltonian. The qualitative differences between the trajectories given by these two types of Hamiltonians was so exciting to Lighthill (1986) that he titled an article in the Proceedings of the Royal Society 'The Recently Recognized Failure of Predictability in Newtonian Dynamics'.

\subsubsection{Two-coordinate Hamiltonians}

When the magnetic field line Hamiltonian has the form $H(x, y)$ at a given point in time, equation (3.1) implies $H$ is constant along the magnetic field lines, $\boldsymbol{B} \cdot \nabla H=0$. Neighbouring trajectories can separate as $e^{\sigma}$ for a Hamiltonian $H(x, y)$ but only in an $\approx e^{-2 \sigma}$ fraction of the area of the $(x, y)$ plane.

The only places at which magnetic field lines can exponentiate apart are at saddle points, where $H(x, y)$ has the Taylor expansion $H=H_{s p}+\left(\partial^{2} H / \partial x \partial y\right)_{s p}\left(x-x_{s p}\right)$ $\left(y-y_{s p}\right)+\cdots$. When $a_{c}$ is the characteristic spatial scale of the function $H(x, y)$. Lines started within a radius $a_{c} e^{-\sigma}$ of the point $\left(x_{s p}, y_{s p}\right)$ can exponentiate $\sigma$ times before being too far from the saddle point to be influenced by it. The fraction of the area in the $(x, y)$ plane in which $\sigma$ exponentiations can take place is therefore $\approx e^{-2 \sigma}$. 


\subsubsection{Three-coordinate Hamiltonians}

As will be shown, when the magnetic field line Hamiltonian has the form $H(x, y, z)$ at a given point in time, the fraction of the area in a constant- $z$ plane occupied by lines that exponentiate apart is generically of order unity.

The trajectory of a magnetic field line can be written as $\boldsymbol{x}\left(x_{s}, y_{s}, \ell\right)$, where $\left(x_{s}, y_{s}\right)$ is the starting point of the trajectory. The distance along the line is $\ell$, which in the reduced MHD model is indistinguishable from $z$. That is,

$$
\begin{gathered}
\boldsymbol{x}\left(x_{s}, y_{s}, \ell\right)=\boldsymbol{x}_{\perp}\left(x_{s}, y_{s}, \ell\right)+\ell \hat{z}, \\
\text { where } \quad \boldsymbol{x}_{\perp}\left(x_{s}, y_{s}, \ell\right) \equiv x\left(x_{s}, y_{s}, \ell\right) \hat{x}+y\left(x_{s}, y_{s}, \ell\right) \hat{y}, \\
x\left(x_{s}, y_{s}, \ell=0\right)=x_{s}, \quad \text { and } y\left(x_{s}, y_{s}, \ell=0\right)=y_{s} .
\end{gathered}
$$

It is convenient to use $\ell$ to indicate the use of $\left(x_{s}, y_{s}, \ell\right)$ as spatial coordinates rather than Cartesian coordinates $(x, y, z)$. The position vector $\boldsymbol{x}\left(x_{s}, y_{s}, \ell\right)$ defines a coordinate system in which the magnetic field lines are trivial.

Three notations can be used for the derivative of a function along a magnetic field line:

$$
\left(\frac{\partial f}{\partial z}\right)_{x_{s} y_{s}}=\frac{\mathrm{d} f}{\mathrm{~d} z}=\frac{\partial f}{\partial \ell}=\frac{\mathrm{d} f}{\mathrm{~d} \ell} .
$$

The total derivative of a function with respect to $z$ means along a magnetic field line as does either the partial or the total derivative with respect to $\ell$.

The separation between neighbouring trajectories is

$$
\begin{gathered}
\boldsymbol{\delta}_{\perp} \equiv \frac{\partial \boldsymbol{x}_{\perp}}{\partial x_{s}} \delta x_{s}+\frac{\partial \boldsymbol{x}_{\perp}}{\partial y_{s}} \delta y_{s}, \\
\text { so }\left(\begin{array}{l}
\delta_{x} \\
\delta_{y}
\end{array}\right)=\left(\begin{array}{ll}
\frac{\partial x}{\partial x_{s}} & \frac{\partial x}{\partial y_{s}} \\
\frac{\partial y}{\partial x_{s}} & \frac{\partial y}{\partial y_{s}}
\end{array}\right)\left(\begin{array}{l}
\delta x_{s} \\
\delta y_{s}
\end{array}\right),
\end{gathered}
$$

where $\boldsymbol{\delta}_{\perp}=\delta_{x} \hat{x}+\delta_{y} \hat{y}$. The Jacobian matrix of $\boldsymbol{x}_{\perp}\left(x_{s}, y_{s}, \ell\right)$ is

$$
\boldsymbol{J}_{\perp} \equiv\left(\begin{array}{ll}
\frac{\partial x}{\partial x_{s}} & \frac{\partial x}{\partial y_{s}} \\
\frac{\partial y}{\partial x_{s}} & \frac{\partial y}{\partial y_{s}}
\end{array}\right)
$$

and the determinant of the Jacobian matrix is the Jacobian $\mathcal{J}_{\perp}$ of the $\left(x_{s}, y_{s}\right)$ coordinates. Since the area element in the $\hat{z}$ direction is $\mathrm{d} \boldsymbol{a}=\hat{z} \mathcal{J}_{\perp} \mathrm{d} x_{s} \mathrm{~d} y_{s}$, magnetic flux conservation implies $\mathcal{J}_{\perp}=1$. The mathematical implication of a unit Jacobian is that the singular value decomposition of the Jacobian matrix has the form

$$
\boldsymbol{J}_{\perp}\left(x_{s}, y_{s}, \ell\right)=\boldsymbol{U}_{\perp} \cdot\left(\begin{array}{cc}
e^{\sigma_{\perp}} & 0 \\
0 & e^{-\sigma_{\perp}}
\end{array}\right) \cdot \boldsymbol{V}_{\perp}^{\dagger},
$$

where $\boldsymbol{U}_{\perp}$ and $\boldsymbol{V}_{\perp}$ are orthogonal matrices. There is a direction in which the magnetic field lines approach each other exponentially, but when integrating lines, numerical 
errors will quickly cause the exponential separation to overwhelm the exponential convergence.

The distance squared between neighbouring magnetic field lines is

$$
\begin{aligned}
\boldsymbol{\delta}_{\perp}^{\dagger} \cdot \boldsymbol{\delta}_{\perp} & =\delta_{x}^{2}+\delta_{y}^{2}=\left(\begin{array}{ll}
\delta x_{s} & \delta y_{s}
\end{array}\right) \cdot \boldsymbol{g} \cdot\left(\begin{array}{l}
\delta x_{s} \\
\delta y_{s}
\end{array}\right) \\
\boldsymbol{g} & \equiv \boldsymbol{J}_{\perp}^{\dagger} \cdot \boldsymbol{J}_{\perp} \\
& =\boldsymbol{V}_{\perp} \cdot\left(\begin{array}{cc}
e^{2 \sigma} & 0 \\
0 & e^{-2 \sigma}
\end{array}\right) \cdot \boldsymbol{V}_{\perp}^{\dagger},
\end{aligned}
$$

where $\boldsymbol{g}$ is the metric tensor.

Equations (3.16) and (3.17) demonstrate that neighbouring magnetic field lines have a separation that depends exponentially on $\sigma_{\perp}\left(x_{s}, y_{s}, \ell\right)$. Neighbouring magnetic fields lines characteristically exponentiate apart with the distance $\ell$ along a line unless there is a constraint that prevents the exponentiation, as when the magnetic field depends on only two spatial coordinates.

Section 2.2 studied the exponentially increasing separation of streamlines $\boldsymbol{x}\left(\boldsymbol{x}_{0}, t\right)$ of the magnetic field line velocity. In the reduced MHD model being considered here, the Jacobian of the streamlines is unity, and the Lyapunov exponents of the streamlines stated as $\sigma(\ell, t)$ and the $\sigma_{\perp}$ of the field lines at a given time can and will be identified.

\subsection{Magnetic field evolution}

The evolution equations for a magnetic field have two parts: (i) the evolution equations for $\boldsymbol{B}$ when the plasma flow velocity $\boldsymbol{v}$ is known and (ii) the evolution equation for the plasma velocity, which will be obtained from force balance in the next section. This section will assume $\boldsymbol{v}$ is known and derive four equations: (i) equation (3.23) relating the time derivative of the Hamiltonian to the streamfunction of the flow, (ii) equation (3.24) relating the Hamiltonian $H$ to the parallel-current function $K \equiv \mu_{0} j_{\|} / B$, (iii) equation (3.26) relating the streamfunction of the flow $\phi$ to the flow vorticity $\Omega$ and (iv) equation (3.27) relating the time derivative of $K$ moving with the flow to the derivative of the vorticity $\Omega$ along a field line.

\subsubsection{Relation between $\partial H / \partial t$ and $\mathrm{d} \phi / \mathrm{d} \ell$}

The time derivative of the magnetic field line Hamiltonian is related to the streamfunction of the flow $\phi$ by Ohm's law and Faraday's law.

The electric field is given by Ohm's law, which will be written as

$$
\begin{aligned}
\boldsymbol{E}+\boldsymbol{v} \times \boldsymbol{B} & =\mathcal{N}_{B} B_{g} \hat{z}, \\
\text { where } \quad \mathcal{N}_{B} & \equiv\left(\frac{c}{\omega_{\mathrm{pe}}}\right)^{2}\left(\frac{\partial}{\partial t}+v_{c}\right) K .
\end{aligned}
$$

$\mathcal{N}_{B}$ represents the non-ideal effects in Ohm's law and hence in the magnetic evolution. The resistivity $\eta$ is related to the electron collision frequency $v_{c}$ by $\eta / \mu_{0}=\left(c / \omega_{\mathrm{pe}}\right)^{2} v_{c}$. The term involving the time derivative of the parallel-current density, where $K \equiv \mu_{0} j_{\|} / B_{g}$, is due to the electron inertia and is always present since the electron is the lightest charged particle. 
Faraday's law implies $\boldsymbol{E}=-\partial \boldsymbol{A} / \partial t-\nabla \Phi$, where the potential $\Phi$ depends on the gauge used to represent the vector potential. When the velocity $v=\nabla \phi \times \hat{z}$, equation (3.2), the clever choice of gauge gives

$$
\boldsymbol{E}=\left(-\frac{\partial H}{\partial t} \hat{z}+\nabla \phi\right) B_{g}
$$

Ohm's law implies, using

$$
\begin{aligned}
\boldsymbol{v} \times \boldsymbol{B} & =(\nabla \phi \times \hat{z}) \times \boldsymbol{B} \\
& =\hat{z} \boldsymbol{B} \cdot \nabla \phi-B_{g} \nabla \phi, \\
\text { that } \frac{\partial H}{\partial t} & =\frac{\mathrm{d} \phi}{\mathrm{d} \ell}-\mathcal{N}_{B} .
\end{aligned}
$$

\subsubsection{Relation between $H$ and $K$}

The relation between the Hamiltonian and the parallel-current function follows simply from Ampere's law, $\nabla \times \boldsymbol{B}=\mu_{0} \boldsymbol{j}$,

$$
\nabla_{\perp}^{2} H=-K
$$

\subsubsection{Relation between $\phi$ and $\Omega$}

The vorticity of the flow is

$$
\begin{aligned}
\Omega & \equiv \hat{z} \cdot \nabla \times \boldsymbol{v} \\
& =-\nabla_{\perp}^{2} \phi,
\end{aligned}
$$

where $\phi$ is the streamfunction.

\subsubsection{Relation between $\mathrm{d} K / \mathrm{d} t$ and $\mathrm{d} \Omega / \mathrm{d} \ell$}

Two equations from the appendix, equations (D 1) relating the total time derivative to the velocity potential and (D4) for the commutator of $d / d t$ and $d / d \ell$, then imply

$$
\frac{\mathrm{d} K}{\mathrm{~d} t}=\frac{\mathrm{d} \Omega}{\mathrm{d} \ell}+\nabla_{\perp}^{2} \mathcal{N}_{B}
$$

The dissipative term $\nabla_{\perp}^{2} \mathcal{N}_{B}$ acts to flatten $K$ across the magnetic field lines.

In an ideal magnetic evolution, $\mathcal{N}_{B}=0$ and $\boldsymbol{v}$ is the velocity of the magnetic field lines. The twisting of the field lines around themselves, given by $\mathrm{d} \Omega / \mathrm{d} \ell$, gives the time rate of change of the current $K$ required to be consistent with both Faraday's and Ampere's law.

\subsubsection{Discussion of $B$ evolution}

The current function $K$ need not be become large to produce a fast magnetic reconnection. As the exponentiation $\sigma$ of neighbouring trajectories becomes larger, $K$ need only increase in proportion to $\sigma$ (Boozer 2012).

The operator $\nabla_{\perp}^{2} \mathcal{N}_{B}$ becomes very large when magnetic field lines exponentiate apart. The theory of general coordinates (see the appendix of Boozer 2004) implies

$$
\nabla_{\perp}^{2} \mathcal{N}_{B}=\frac{1}{\mathcal{J}_{\perp}} \frac{\partial}{\partial \boldsymbol{x}_{s}} \cdot\left(\mathcal{J}_{\perp} \boldsymbol{g}^{-1} \cdot \frac{\partial \mathcal{N}_{B}}{\partial \boldsymbol{x}_{s}}\right)
$$




$$
\boldsymbol{g}^{-1}=\boldsymbol{V}_{\perp} \cdot\left(\begin{array}{cc}
e^{-2 \sigma} & 0 \\
0 & e^{2 \sigma}
\end{array}\right) \cdot \boldsymbol{V}_{\perp}^{\dagger} \text {. }
$$

Although the operator $\nabla_{\perp}^{2}$ can be calculated in the field line coordinates $\left(x_{s}, y_{s}, \ell\right)$, equation (3.28), this operator is easier to calculate and to invert in ordinary Cartesian coordinates $(x, y, z)$.

To determine the evolution of the magnetic field lines $\boldsymbol{x}_{\perp}\left(x_{s}, y_{s}, z, t\right)$, the Hamiltonian $H(x, y, z, t)$ is required at each time $t . H(x, y, z, t)$ can be obtained from $K\left(x_{s}, y_{s}, z, t\right)$ by use of the Green's function for a two-dimensional Laplacian:

$$
H=\int K \frac{\ln \left(\left(x-x^{\prime}\right)^{2}+\left(y-y^{\prime}\right)^{2}\right)}{4 \pi} \mathrm{d} x_{s} \mathrm{~d} y_{s},
$$

where $K, x^{\prime}$, and $y^{\prime}$ are written as functions of $\left(x_{s}, y_{s}, z, t\right)$. The Jacobian between $\left(x_{s}, y_{s}\right)$ and $(x, y)$ coordinates is unity, so $\mathrm{d} x^{\prime} \mathrm{d} y^{\prime}=\mathrm{d} x_{s} \mathrm{~d} y_{s} . H(x, y, z, t)$ can also be obtained by Fourier decomposition:

$$
\begin{aligned}
H & =\int \frac{\tilde{K}\left(k_{x}, k_{y}, z, t\right)}{k_{x}^{2}+k_{y}^{2}} \mathrm{e}^{\mathrm{i}\left(k_{x} x+k_{y} y\right)} \mathrm{d} k_{x} \mathrm{~d} k_{y} \\
\tilde{K} & =\frac{1}{(2 \pi)^{2}} \int K \mathrm{e}^{-\mathrm{i}\left(k_{x} x+k_{y} y\right)} \mathrm{d} x \mathrm{~d} y \\
& =\frac{1}{(2 \pi)^{2}} \int K \mathrm{e}^{-\mathrm{i}\left(k_{x} x+k_{y} y\right)} \mathrm{d} x_{s} \mathrm{~d} y_{s},
\end{aligned}
$$

where $K, x$, and $y$ in the last integral are written as functions of $\left(x_{s}, y_{s}, z, t\right)$. A simple version of the reduced MHD model is periodic in the $x$ and $y$ directions with a periodicity length $2 \pi a$, so $k_{x}=m /(2 \pi a)$ and $k_{y}=n /(2 \pi a)$ with $m$ and $n$ integers.

Equation (3.31) implies the smallest wavenumbers $\boldsymbol{k}_{\perp}=k_{x} \hat{x}+k_{y} \hat{y}$ largely determine $H(x, y, z, t)$. As discussed in $\S 2.1$, the smallest wavenumber terms in $H$ produce the largest separations between magnetic field lines and, therefore, have the largest effect on magnetic reconnection. There is nothing in the model that rules out short wavelength turbulence. Indeed, wavelengths of order $a_{c} e^{-\sigma}$ are strongly driven, and their effect on reconnection is an important question.

\subsection{Force balance}

General features of magnetic reconnection can be studied by considering the generic properties of a flow. This was done in $\S \S 1$ and 2 and could be done using the equations derived in $\S 3.3$. To actually know the flow and the evolution of the magnetic field, an additional equation is required, which is given by force balance. The required (3.40), which relates $\mathrm{d} \Omega / \mathrm{d} t$ to $\mathrm{d} K / \mathrm{d} \ell$ will be derived in this section.

The smallness of the Debye length implies the current must be divergence free (Boozer 2015). That and the expression for the electromagnetic or Lorentz force $\boldsymbol{f}=$ $\boldsymbol{j} \times \boldsymbol{B}$ implies

$$
\begin{aligned}
\boldsymbol{B} \cdot \nabla \frac{j_{\|}}{B} & =\boldsymbol{B} \cdot \nabla \times \frac{\boldsymbol{f}}{B^{2}}, \\
\text { or } \frac{\mathrm{d} K}{\mathrm{~d} \ell} & =\frac{1}{V_{A}^{2}} \hat{z} \cdot \nabla \times \frac{\boldsymbol{f}}{\rho_{0}} ;
\end{aligned}
$$




$$
V_{A}^{2} \equiv \frac{B_{g}^{2}}{\mu_{0} \rho_{0}}
$$

where $\rho_{0}$ is the plasma density, which is assumed to be a constant, and $V_{A}$ is the Alfvén velocity.

The force exerted by the plasma consists of inertial and viscosity terms,

$$
\begin{aligned}
\frac{\boldsymbol{f}}{\rho_{0}} & =\frac{\partial \boldsymbol{v}}{\partial t}+\boldsymbol{v} \cdot \nabla \boldsymbol{v}-v_{v} \nabla_{\perp}^{2} \boldsymbol{v}, \\
\text { and } \boldsymbol{v} \cdot \nabla \boldsymbol{v} & =\nabla\left(v^{2} / 2\right)-\boldsymbol{v} \times \nabla \times \boldsymbol{v}, \\
\text { so } \hat{z} \cdot \nabla \times \frac{\boldsymbol{f}}{\rho_{0}} & =\frac{\mathrm{d} \Omega}{\mathrm{d} t}-v_{v} \nabla_{\perp}^{2} \Omega .
\end{aligned}
$$

Consequently, force balance implies

$$
\begin{aligned}
\frac{\mathrm{d} K}{\mathrm{~d} \ell} & =\frac{1}{V_{A}^{2}}\left(\frac{\mathrm{d} \Omega}{\mathrm{d} t}-\mathcal{N}_{v}\right), \\
\text { where } \quad \mathcal{N}_{v} & \equiv v_{v} \nabla_{\perp}^{2} \Omega
\end{aligned}
$$

gives the non-ideality of the plasma flow.

\subsection{Ideal evolution}

The evolution in the absence of dissipation, $\mathcal{N}_{B}=0$ and $\mathcal{N}_{v}=0$, is given by an Alfvén wave equation, equation (3.45). The ideal equations are

$$
\begin{aligned}
\nabla_{\perp}^{2} H & =-K \\
\frac{\mathrm{d} K}{\mathrm{~d} t} & =\frac{\mathrm{d} \Omega}{\mathrm{d} \ell} \\
\frac{\mathrm{d} K}{\mathrm{~d} \ell} & =\frac{1}{V_{A}^{2}} \frac{\mathrm{d} \Omega}{\mathrm{d} t} .
\end{aligned}
$$

When the evolution is ideal, $\mathrm{d} / \mathrm{d} \ell$ and $\mathrm{d} / \mathrm{d} t$ commute, see equation (D 8). In the ideal case, being fixed to a fluid point, as in a $\mathrm{d} / \mathrm{d} t$ derivative, or fixed on a magnetic field line, as in a $\mathrm{d} / \mathrm{d} \ell$ derivative, is the same. The implication is

$$
\frac{\mathrm{d}^{2} \Omega}{\mathrm{d} t^{2}}=V_{A}^{2} \frac{\mathrm{d}^{2} \Omega}{\mathrm{d} \ell^{2}} .
$$

Equation (3.45) is an equation for Alfvén waves along a magnetic field line that is defined by its position $\left(x_{s}, y_{s}\right)$ at $\ell=0$. Since the field lines do not break, $\left(x_{s}, y_{s}\right)$ is also the position of a fluid point in the upper boundary $z=L$, which means a solution $x\left(x_{s}, y_{s}, t\right), y\left(x_{s}, y_{s}, t\right)$ to the equations $\mathrm{d} x / \mathrm{d} t=\partial \phi_{w} / \partial y$ and $\mathrm{d} y / \mathrm{d} t=-\partial \phi_{w} / \partial x$ with the initial condition $x\left(x_{s}, y_{s}, t=0\right)=x_{s}$ and $y\left(x_{s}, y_{s}, t=0\right)=y_{s}$. Conventionally, a wave equation is written using partial rather than total derivatives. This can be done by noting $\mathrm{d} / \mathrm{d} t=(\partial / \partial t)_{x_{s}, y_{s}}$ and $\mathrm{d} / \mathrm{d} \ell=(\partial / \partial \ell)_{x_{s}, y_{s}}$. 


\subsubsection{General Alfvén wave solution}

Along each magnetic field line, which means for each $\left(x_{s}, y_{s}\right)$, the general solution to equation (3.45) is a sum of two functions of one variable

$$
\Omega(\ell, t)=\Omega_{d}\left(t+\frac{\ell-L}{V_{A}}\right)+\Omega_{u}\left(t-\frac{\ell}{V_{A}}\right),
$$

where $\Omega_{d}$ represents a downward going and $\Omega_{u}$ an upward going Alfvén wave. The two boundary conditions that determine $\Omega_{d}$ and $\Omega_{u}$ are $\Omega(z=0, t)=0$ and $\Omega(L, t)=$ $\Omega_{w}^{(s)}(t)$, where $\Omega_{w}^{(s)}(t)$ is the vorticity of the flow in the wall at the location given by $\left(x_{s}, y_{s}\right)$ at $\ell=0$. The vorticity of the flow in the wall is $\Omega_{w}(x, y, t)=-\nabla^{2} \phi_{w}(x, y, t)$, and $\Omega_{w}^{(s)}(t)=\Omega_{w}\left(x\left(x_{s}, y_{s}, t\right), y\left(x_{s}, y_{s}, t\right), t\right)$.

The solution assuming the flow in the wall is zero for $t<0$ is

$$
\begin{aligned}
& \Omega_{d}(t)=\Omega_{w}^{(s)}(t) \quad \text { for } t<\frac{2 L}{V_{A}} \\
& \Omega_{d}(t)=\Omega_{w}^{(s)}(t)+\Omega_{d}\left(t-\frac{2 L}{V_{A}}\right) \quad \text { for } t>\frac{2 L}{V_{A}} \\
& \Omega_{u}(t)=-\Omega_{d}\left(t-\frac{L}{V_{A}}\right) \quad \text { for } t>\frac{L}{V_{A}} .
\end{aligned}
$$

When the vorticity of the wall flow evolves slowly compared to the Alfvén transit time, $\tau_{A} \equiv L / V_{A}$ :

$$
\begin{aligned}
\frac{\mathrm{d} \Omega_{d}(t)}{\mathrm{d} t} & =\frac{\Omega_{w}^{(s)}(t)}{2 \tau_{A}} \\
\Omega_{u}(t) & =-\Omega_{d}(t)+\frac{\Omega_{w}^{(s)}(t)}{2} \\
\Omega(\ell, t) & =\Omega_{w}^{(s)}(t) \frac{\ell}{L} \\
\frac{\mathrm{d} K}{\mathrm{~d} t} & =\frac{\Omega_{w}^{(s)}(t)}{L} .
\end{aligned}
$$

\subsubsection{Discussion of ideal solution and its breaking}

The function $\Omega_{w}^{(s)}(t)$ is purely determined by the properties of the flow in the perfectly conducting wall at $z=L$. Once $\Omega_{w}^{(s)}(t)$ is known, a solution for $K\left(x_{s}, y_{s}, \ell, t\right)$ is determined, essentially analytically, by (3.46) to (3.49). The solution for the magnetic field lines $\boldsymbol{x}_{\perp}\left(x_{s}, y_{s}, z, t\right)$ in the region $0<z<L$ requires the Hamiltonian $H(x, y, z, t)$. The direct solution for Hamiltonian $H_{d}(x, y, z)$, which can be obtained from $K\left(x_{s}, y_{s}, \ell\right)$ using (3.30) to (3.33), will not be exact and small adjustments will generally be required for the field lines started at $\left(x_{s}, y_{s}\right)$ to reach the required positions at $z=L$. One way to do this is to let $H(x, y, z)=H_{d}(x, y, z)+\delta H(x, y)$. The function $\delta H$ has sufficient freedom to achieve an exact match of the field lines between their starting and ending position. $K$ is modified by $\delta H$, but presumably the modification is small when a solution is accurately calculated.

This slowly evolving solution need not be the only solution. Indeed it is not unique when the system has an ideal instability (Huang, Bhattacharjee \& Boozer 2014), which allows for a change in the magnetic configuration on a time scale set by the Alfvén 
transit time $L / V_{A}$. The importance of ideal instabilities in fast magnetic reconnection needs to be better understood.

As discussed in $\S \S 1$ and 2, the properties of the ideal solution determine a large part of the theory of fast magnetic reconnection - even when non-ideal effects become important. The ideality of the magnetic evolution, when expressed by the relation $\mathrm{d} K / \mathrm{d} t=\mathrm{d} \Omega / \mathrm{d} \ell+\nabla_{\perp}^{2} \mathcal{N}_{B}$, is broken when the diffusive term $\nabla_{\perp}^{2} \mathcal{N}_{B}$ becomes comparable to $\mathrm{d} K / \mathrm{d} t$ for terms in $K$ that have a small $\boldsymbol{k}_{\perp}$. Alfvén speed relaxation is modified by viscosity when the diffusive term for the vorticity $\nu_{v} \nabla_{\perp}^{2} \Omega$ has a similar relation to small $\boldsymbol{k}_{\perp}$ terms in $\Omega$. The complexity of the geometry when $\sigma$ is large implies the $\nabla_{\perp}^{2}$ operator becomes large indeed, equation (3.28), even when the streamlines in the flowing boundary do not themselves exponentiate apart, as when $\phi_{w}$ is independent of time. The complexity is even greater when the streamlines do exponentiate apart, and it is important to understand the effect of streamline exponentiation on reconnection.

\subsection{Dimensionless ratios}

The drive for reconnection in the simple model of $\S 3$ is the flow $\boldsymbol{v}_{w}=\nabla \phi_{w} \times \hat{z}$ in the wall, which is often assumed to be very slow

$$
M_{e f f} \equiv \frac{v_{w} L}{V_{A} a_{c}} \ll 1,
$$

where $a_{c}$ is the characteristic spatial scale for variations in $\phi_{w}$.

By definition, an ideal magnetic evolution can move the magnetic field lines but cannot break them. The expression for the electric field has two terms that break the ideal evolution of the magnetic field. The dimensionless coefficients that give the strength of this breaking are the magnetic Reynolds number,

$$
\begin{aligned}
& R_{m} \equiv \frac{a_{c} v_{w}}{\eta / \mu_{0}}, \\
& \text { and } \frac{a_{c}}{c / \omega_{\mathrm{pe}}}
\end{aligned}
$$

Both dimensionless coefficients must be very large compared to unity to have a nontrivial reconnection problem.

The viscosity $v_{v}$ breaks the ideal equation for the plasma flow and has the Reynolds number as its dimensionless coefficient,

$$
R_{e} \equiv \frac{a_{c} v_{w}}{v_{v}} .
$$

A non-trivial problem in magnetic reconnection exists for any value of $R_{e}$. The strength of the viscosity is sometimes given by the magnetic Prandtl number $P_{m} \equiv \mu_{0} v_{v} / \eta$, which can be very large compared to unity in plasmas. A more important parameter in measuring the relative importance of viscous to resistive dissipation will be found to be the Alfvén-weighted Prandtl number,

$$
\begin{aligned}
\mathcal{P}_{A} & \equiv M_{e f f}^{2} P_{m} \\
& =\left(\frac{v_{w} L}{a_{c} V_{A}}\right)^{2} \frac{\mu_{0} v_{v}}{\eta} .
\end{aligned}
$$




\subsection{Alfvén waves with dissipation}

When the magnetic field evolution is non-ideal, there is a slippage between the magnetic field lines and the plasma, so $\mathrm{d} / \mathrm{d} \ell$ and $\mathrm{d} / \mathrm{d} t$ no longer commute. Equation (D 8) implies

$$
\frac{\mathrm{d}}{\mathrm{d} t} \frac{\mathrm{d} K}{\mathrm{~d} \ell}=\frac{\mathrm{d}}{\mathrm{d} \ell} \frac{\mathrm{d} K}{\mathrm{~d} t}-\hat{z} \cdot\left(\nabla_{\perp} K \times \nabla_{\perp} \mathcal{N}_{B}\right) .
$$

Equations (3.27) and (3.40) then give the equation for Alfvén waves with dissipation

$$
\begin{aligned}
\frac{\partial^{2} \Omega}{\partial \ell^{2}} & =\frac{1}{V_{A}^{2}} \frac{\mathrm{d}^{2} \Omega}{\mathrm{d} t^{2}}+\mathcal{N}_{A} \\
\mathcal{N}_{A} & \equiv-\frac{\nu_{v}}{V_{A}^{2}} \frac{\mathrm{d}}{\mathrm{d} t} \nabla_{\perp}^{2} \Omega-\frac{\mathrm{d} \nabla_{\perp}^{2} \mathcal{N}_{B}}{\mathrm{~d} \ell}+\hat{z} \cdot\left(\nabla_{\perp} K \times \nabla_{\perp} \mathcal{N}_{B}\right) .
\end{aligned}
$$

The two dissipative terms, one proportional to $v_{v}$ and the other proportional to $\eta / \mu_{0}$, have a ratio of the Alfvén-weighted Prandtl number $\mathcal{P}_{A}$, equation (3.58), which follows from $H \sim a_{c}^{2} / L$ and $\Omega \sim v_{w} / a_{c}$. The term $\mathrm{d}^{2} \Omega / \mathrm{d} t^{2}$ has a relative size comparable to the viscosity term of the Reynold number $R_{e}$.

When the viscosity dominates over resistivity in the damping of Alfven waves, equation (3.60) can be written in a particularly simple form

$$
\frac{\mathrm{d}}{\mathrm{d} t}\left(\frac{\mathrm{d} \Omega}{\mathrm{d} t}-\mathcal{N}_{v}\right)=V_{A}^{2} \frac{\partial^{2} \Omega}{\partial \ell^{2}}
$$

\subsection{Difficulty of obtaining solutions}

The evolution of the model for a driven magnetic field can be obtained by integration until the number of exponentiations $\sigma$ in the separation between neighbouring magnetic field lines becomes large. Unfortunately, the difficulty of following the evolution increases as $e^{5 \sigma}$, so a hard cutoff exists in the maximum value of $\sigma$ that can be resolved. To follow the evolution, high numerical precision is required; numerical errors blur the evolution of magnetic field lines as does $c / \omega_{\text {pe }}$.

A petascale computer can perform $10^{15}$ operations a second or $\approx 10^{26}$ per day. When $\sigma=10$, the number of operations is increased by approximately $10^{22}$ times over the case when $\sigma \lesssim 1$, so $\sigma \approx 10$ appears to be an upper limit on what can be computed. Increasing the computer power by a thousand increases the maximum computable $\sigma$ from $\sigma \approx 10$ to $\sigma \approx 11.4$.

Reaching values of $\sigma \approx 8$ for studying magnetic reconnection in fusion devices is credible, but $\sigma \approx 20$, which is required to understand reconnection in the corona, appears impossible. Simulations using modest values of $\sigma$ must be sufficiently well understood to devise extrapolations or reliable approximations.

Once $\sigma$ becomes large, distances in the $(x, y)$ plane of order $a_{c} e^{-\sigma}$ must be resolved as must distances of order $L e^{-\sigma}$ in the $z$ direction. The speed with which the magnetic field lines move is of order $v_{w} e^{\sigma}$, so the time required for a field line to move over a spatial scale $a_{c} e^{-\sigma}$ is $\left(a_{c} / v_{w}\right) e^{-2 \sigma}$. Assuming the computational difficulty scales as the number of spatial grid cells times the number of time steps, the difficulty scales as $e^{5 \sigma}$. 


\section{Discussion}

When dissipation is low, Alfvénic reconnection is so prevalent that it must be a natural consequence of an ideal evolution. The most important result of the paper is the proof that this is indeed the case, $\S 2$.

The sixty-year focus on two-coordinate models to represent magnetic reconnection in three-dimensional space has obscured physics. The generic tendency for evolving magnetic fields to reach states with Alfvén speed reconnection is eliminated by restricting the evolution to a two-coordinate space. Two-coordinate models of magnetic reconnection may be adequate to describe some features of reconnection, but are obviously only an approximation in three-dimensional space. The range of validity of two-dimensional models can only be established by derivations that begin with all three spatial dimensions.

The most important effect eliminated by a two-dimensional approximation is the exponentially large distortion of infinitesimal magnetic flux tubes with distance along the tube. As shown in $\$ 2.1$, the exponential enhancement of reconnection in three spatial dimensions is analogous to the increase in mixing of incompressible fluids produced by time depending stirring in two-dimensional models.

The absence of studies of how ideally evolving magnetic fields go from smooth initial to Alfvénic reconnecting states makes simple models of profound importance. Even heuristic arguments, such as those given in the introduction and $\S$ C.3, show that any star with evolving magnetic fields in the photosphere on a much longer spatial scale than a gravitational scale height must have a corona. Calculations could clarify whether this explains the height of the transition region and the electron energy in the corona of the Sun.

Many questions on driven reconnection that remain unanswered could be answered in the driven reconnection model derived in $\S 3$. This model is based on a paper by van Ballegooijen (1985). The equations have been studied by a number of authors, notably Ng et al. (2012) and Huang et al. (2014). The study by Huang et al is closely related to the model discussed here but was limited to exponentiations $\sigma \lesssim 6$. Daughton et al. (2014) have done extensive kinetic calculations of magnetic reconnection in a more complete magnetic field model retaining all three spatial coordinates, but a Harris sheet was used as an initial condition, and the number of exponentiations was $\sigma \lesssim 8$. No studies have been done that follow a smooth initial into a rapidly reconnecting state - even the ideal part of that evolution has not been followed, which as shown in $\$ 3.5$, can be greatly simplified by analytic methods.

A number of questions need to be addressed and many could be within the model given in $\S 3$.

(i) How does the parallel-current density increase as the system evolves?

The parallel-current density is the part that directly affects reconnection and its increase is determined by (3.27). Although reconnection generally reaches an Alfvénic rate within the reconnection model of this paper, it appears difficult to produce a singular current density.

(ii) How does the reconnection depend on the three dimensionless parameters $R_{m}$, $\mathcal{P}_{A}$ and $M_{\text {eff }}$ ?

Reconnection is trivial unless the magnetic Reynolds number $R_{m}$ is very large compared to unity, but the complexity of the behaviour as $R_{m} \rightarrow \infty$ is poorly understood. Unless the plasma has significant dissipation, the energy released by the reconnection will drive strong Alfvén waves. These waves propagate along the magnetic field lines and develop an extremely complicated spatial dependence and 
enhanced dissipation from exponentially increasing separation of the lines (Similon \& Sudan 1989).

Significant dissipation with a very large magnetic Reynolds number requires the Alfvén-weighted Prandtl number $\mathcal{P}_{A}$ be large. The dependence of reconnection phenomena on $\mathcal{P}_{A}$ is not understood. Although the Alfvénic Mach number $M_{\text {eff }}$ is generally assumed to be small compared to unity, the actual plasma flow can become large due to the large spatial excursions made by magnetic field lines. Indeed, the plasma may become unstable (Huang et al. 2014) and make a transition to a different state on an Alfvénic time scale.

(iii) How does the reconnection depend on the complexity of the drive $\phi_{w}(x, y, t)$ ?

An important but poorly understood question is, when the drive $\phi_{w}$ continues for a long time, does the reconnection settle into a quasi-steady state or is it episodic? A finite time, the trigger time, is clearly required to obtain the first reconnection event when the initial condition is $H=0$. The dependence of the trigger time on either the dimensionless parameters or the complexity of $\phi_{w}$ is essentially unknown.

Is the reconnection behaviour qualitatively similar for all functions $\phi_{w}(x, y, t)$ that have a similar characteristic spatial scale $a_{c}$ ? When $\phi_{w}(x, y, t)$ depends on time, neighbouring points in the flowing wall generically separate exponentially in time; when $\phi_{w}$ has no time dependence neighbouring wall points do not exponentiate apart. Does this produce a qualitative difference in the reconnection?

When the dominant spatial scale $a_{c}$ of $\phi_{w}$ is small compared to the size $a$ of the reconnecting region, which may be a periodicity length $2 \pi a$, the reconnection has a relatively slow diffusive nature, but this is not well understood.

(iv) Do the short wavelength structures that are driven by fast reconnection in three dimensions play an important role by modifying the timing and the nature of the breaking of the ideal constraints?

When neighbouring field lines exponentiate apart by a factor $e^{\sigma}$, structures are strongly driven on the scale $a_{c} e^{-\sigma}$.

(v) How quickly do plasma elements that originally lay along one field line spread across the reconnecting volume?

The rapid spreading of impurities across a tokamak plasma during a disruption is poorly understood but may be a by-product of the fast magnetic reconnection that is associated with the current spike (Boozer 2017).

(vi) Under what conditions does $c / \omega_{\mathrm{pe}}$ dominate the resistivity $\eta$ in causing magnetic field line breaking?

The large magnetic field line excursions that occur when $\sigma$ is large can give large flow velocities and make the time derivative term involving $c / \omega_{\mathrm{pe}}$ far more important than it appears to be.

(vii) When $\phi_{w}$ has a form that gives episodic rather than quasi-steady-state reconnection, how quickly is equilibrium, $\mathrm{d} K / \mathrm{d} \ell=0$, re-established?

This question is of particular interest in tokamak disruptions because it presumably determines the duration of the current spike (Boozer 2017).

(viii) How is the plasma heating spread over the plasma?

When the magnetic Reynolds number $R_{m}$ is very large, reconnection can occur with little dissipation of energy; most of the energy goes first into Alfvén waves. The rapidity with which the Alfvén waves are damped is a complicated issue (Similon \& Sudan 1989; Boozer 2014) but determines both the spatial region and the plasma species that is heated. The results of this paper imply the development of current sheets as described in Boozer (2014) is incorrect, but the damping of Alfvén waves in nonetheless strong because of the large increase in $\nabla_{\perp}^{2} \Omega$ due to magnetic field line exponentiation. 


\section{Acknowledgements}

This material is based upon work supported by the U.S. Department of Energy, Office of Science, Office of Fusion Energy Sciences under award nos DE-FG0295ER54333 and DE-FG02-03ER54696.

\section{Appendix A. Plasma versus magnetic field line velocity}

The small gyroradius Ohm's law, $\boldsymbol{E}+\boldsymbol{v} \times \boldsymbol{B}=\eta_{\perp} \boldsymbol{j}_{\perp}+\eta_{\|} \boldsymbol{j}_{\|}$, illustrates the importance of distinguishing between the plasma velocity $\boldsymbol{v}$ and the magnetic field line velocity $\boldsymbol{u}$. This Ohm's law can be written as $\boldsymbol{E}+\boldsymbol{u} \times \boldsymbol{B}=\eta_{\| \mid} \boldsymbol{j}_{\|}$with $\boldsymbol{u}=\boldsymbol{v}+\eta_{\perp} \boldsymbol{j}_{\perp} \times \boldsymbol{B} / B^{2}$. The velocity of the plasma $\boldsymbol{v}$ is the sum of the velocity of the magnetic field lines and the velocity of the plasma across the lines $-\eta_{\perp} \boldsymbol{j}_{\perp} \times \boldsymbol{B} / B^{2}$. Letting $\boldsymbol{j} \times \boldsymbol{B}=\nabla p$ and ignoring the temperature gradient, the plasma velocity across the magnetic field lines is $\boldsymbol{v}-\boldsymbol{u}=-D_{c l} \nabla n$. The classical diffusion coefficient $D_{c l} \equiv \eta_{\perp} n T / B^{2}$ can be written as $D_{c l}=\left(m_{e} / m_{i}\right) \rho_{i}^{2} v_{c}$, where $\rho_{i}$ is the ion gyroradius and $v_{c}$ is the electron collision frequency in $\eta_{\perp}$. In large tokamaks, microturbulence causes particle transport that is of order gyro-Bohm diffusion $D_{g B}=\left(\rho_{i} / R_{0}\right)(T / e B)$, where $R_{0}$ is the major radius. The ratio of the observed to the classical particle transport, $D_{g B} / D_{c l}=\left(v_{i} / R_{0}\right) /\left(m_{e} \tau_{e} / m_{i}\right) \approx 10^{5}$, where $v_{i}$ is the ion thermal speed, so the effective $\eta_{\perp}$ is enhanced by a factor $\approx 10^{5}$ over its classical value. Boozer (2004) has shown slippage of the poloidal compared to the toroidal magnetic flux in tokamaks, which is given by the magnetic field line velocity $\boldsymbol{u}$ or equivalently the loop voltage, is determined by $\eta_{||} j_{\|}$. The observed parallel resistivity, when corrected by approximately a factor of two for trapped particle effects, is consistent with the classical value of $\eta_{\|}$. In other words, in large tokamak experiments $\eta_{\perp} / \eta_{\|} \approx 10^{5}$.

\section{Appendix B. Relativistic extensions}

The Lorentz transformation for the electric and magnetic fields demonstrates that both the concept of a magnetic field line velocity $\boldsymbol{u}$ and the anti-reconnection theorem remain valid and important in relativistic theory. Jackson (1998) in his equation (11.149) gave this Lorentz transformation. When the velocity $\boldsymbol{u}$ is perpendicular to both $\boldsymbol{E}$ and $\boldsymbol{B}$, Jackson's equation implies

$$
\begin{aligned}
& \boldsymbol{E}_{u}=\gamma(\boldsymbol{E}+\boldsymbol{u} \times \boldsymbol{B}) ; \\
& \boldsymbol{B}_{u}=\gamma\left(\boldsymbol{B}-\frac{1}{c^{2}} \boldsymbol{u} \times \boldsymbol{E}\right) .
\end{aligned}
$$

Let $\boldsymbol{E}(\boldsymbol{x}, t)$ and $\boldsymbol{B}(\boldsymbol{x}, t)$ be the electric and magnetic fields in the frame of reference that is the basis of the analysis. The evolution of the magnetic field in that frame, $\partial \boldsymbol{B} / \partial t=-\boldsymbol{\nabla} \times \boldsymbol{E}$, is identical to that in a plasma in which the electric field is $\mathcal{E}=$ $\boldsymbol{E}+\nabla \Phi$. The electric field $\mathcal{E}$ will be the basis of the analysis, and the freedom of $\Phi$ will be used to make $\mathcal{E} \cdot \boldsymbol{B}=0$, at least locally. Since $\mathcal{E} \cdot \boldsymbol{B}$ is a Lorentz invariant, the transformed quantity $\mathcal{E}_{u} \cdot \boldsymbol{B}_{u}=0$. In the frame moving with a velocity $\boldsymbol{u}=\mathcal{E} \times \boldsymbol{B} / B^{2}$ relative to the first, the perpendicular electric field is zero, $\mathcal{E}_{u} \times \boldsymbol{B}_{u}=0$, so in that frame $\mathcal{E}_{u}=0$, and the magnetic field $\boldsymbol{B}_{u}$ does not evolve. Letting $\beta \equiv|\boldsymbol{u}| / c$, one finds that the requirement to make $\mathcal{E}_{u} \times \boldsymbol{B}_{u}=0$ is $\beta /\left(1+\beta^{2}\right)=c \mathcal{E} B /\left(\mathcal{E}^{2}+c^{2} B^{2}\right)$. A frame in which there is no electric field is only possible when $\beta=\mathcal{E} / c B<1$. When $\mathcal{E} / c B>$ 1 with $\mathcal{E} \cdot \boldsymbol{B}=0$, there is no magnetic field in a frame moving with velocity $\boldsymbol{u}=$ $c^{2} \mathcal{E} \times \boldsymbol{B} / \mathcal{E}^{2}$. The Lorentz invariant $B^{2}-\mathcal{E}^{2} / c^{2}$ ensures distinct behaviour depending on whether $\mathcal{E} / c B$ is greater or less than unity. 


\section{Appendix C. Spherical geometry}

\section{C.1. Curl-free magnetic fields}

The sphere is the only smooth and bounded shape that has simple solutions to Laplace's equation, $\nabla^{2} \phi=0$. In spherical geometry a curl-free magnetic field has the form

$$
\begin{aligned}
\boldsymbol{B} & =\nabla g \\
g & =\sum_{\ell n} \frac{a B_{\ell}^{n}}{\ell}\left(\frac{r}{a}\right)^{\ell} Y_{\ell}^{n}(\theta, \varphi), \quad r<a \\
& =-\sum_{\ell n} \frac{a B_{\ell}^{n}}{\ell+1}\left(\frac{a}{r}\right)^{\ell+1} Y_{\ell}^{n}(\theta, \varphi), \quad r>a,
\end{aligned}
$$

where the $Y_{\ell}^{n}$ are the spherical harmonics. The coefficient $B_{\ell}^{n}$ is uniquely determined by the $n \ell$ normal component $B_{n}(\theta, \varphi)$ of the magnetic field on the $r=a$ surface of the sphere,

$$
B_{n}(\theta, \varphi)=\sum_{\ell n} B_{\ell}^{n} Y_{\ell}^{n}(\theta, \varphi)
$$

When the magnetic field is symmetric about the axis of the sphere, $\varphi$ symmetry, only $n=0$ spherical harmonics appear and the vector potential for the magnetic field is

$$
\begin{aligned}
\boldsymbol{A} & =\psi \nabla \frac{\varphi}{2 \pi} ; \\
\nabla \frac{\varphi}{2 \pi} & =\frac{\hat{\varphi}}{2 \pi r \sin \theta} ; \\
\boldsymbol{B} & =\nabla \psi \times \nabla \frac{\varphi}{2 \pi} .
\end{aligned}
$$

The magnetic field lines lie in a constant $\psi$ and constant $\varphi$ plane. The magnetic flux is $\psi=\oint \boldsymbol{A} \cdot(\partial \boldsymbol{x} / \partial \varphi) \mathrm{d} \varphi$. Letting $Y_{\ell}(\theta) \equiv Y_{\ell}^{n=0}$ and $B_{\ell} \equiv B_{\ell}^{0}$, then one finds by equating the magnetic field derived from $g$ and from $\psi$,

$$
\begin{aligned}
\psi & =\sum_{\ell} \psi_{\ell}\left(\frac{r}{a}\right)^{\ell+1} G_{\ell}(\theta) ; \\
\psi_{\ell} & =2 \pi a^{2} B_{\ell} ; \\
G_{\ell}(\theta) & =-\frac{\sin \theta}{\ell(\ell+1)} \frac{\mathrm{d} Y_{\ell}}{\mathrm{d} \theta} \\
\frac{\mathrm{d} G_{\ell}}{\mathrm{d} \theta} & =\sin \theta Y_{\ell} .
\end{aligned}
$$

\section{C.2. Magnetic nulls}

This section will show that magnetic field lines from one magnetic field null, $B^{2}=0$ need not strike another null in a short distance even when a second null lies nearby and that magnetic nulls are generically rare in space.

First, consider the rarity of nulls in space. Using Cartesian coordinates, a magnetic null implies the solution of three equations with three unknowns: $B_{x}(x, y, z)=0$, 
$B_{y}(x, y, z)=0$ and $B_{z}(x, y, z)=0$. Three equations with three unknowns are well known to generically have solutions that are discrete if any solutions exist at all. The standard counterexample is a field with perfect symmetry, such a $z$-symmetry, which means $B_{x}, B_{y}$ and $B_{z}$ are independent of $z$. When all three components vanish at $x_{0}, y_{0}$, then there is a line null at $\left(x_{0}, y_{0}\right)$ for all $z$. The addition of an arbitrarily small $z$-dependent field will break the line null into discrete point nulls. For example, $\delta B_{x} \propto \sin z$ will break the line null into point nulls, one at $\left(x_{0}, y_{0}, z=0\right)$ and another at $\left(x_{0}, y_{0}, z=\pi\right)$, no matter how small the amplitude of $\delta B_{x}$.

Second, consider the existence of field lines that connect nulls. When a null exists in a locally curl-free magnetic field, then one can locate a sphere about the null and obtain an isolated null by the magnetic potential $g=\left(a B_{2}\right) / 2(r / a)^{2} Y_{2}(\theta)$, where $Y_{2} \propto \cos ^{2} \theta-1 / 3$, the magnetic field has a single null at $r=0$ and the flux $\psi=0$. Note $B_{r} \propto r\left(\cos ^{2} \theta-1 / 3\right)$ and $B_{\theta} \propto r \sin 2 \theta$. One could include $Y_{2}^{n}$ harmonic terms as well, for example $Y_{2}^{2} \propto \sin ^{2} \theta \sin (2 \varphi)$, but when they are small they introduce no new nulls in the region $r<a$ in which the magnetic field is curl free and would make $\psi$ conservation not rigorously valid.

A field with one additional null can be produced, preserving symmetry in $\varphi$, by adding a term to the magnetic potential $\propto r^{3} Y_{3}(\theta)$, where $Y_{3} \propto \cos ^{3} \theta-(3 / 5) \cos \theta$. That is,

$$
\begin{aligned}
& g=\frac{a B_{2}}{2}\left(\frac{r}{a}\right)^{2}\left(\cos ^{2} \theta-\frac{1}{3}\right)+\frac{a B_{3}}{3}\left(\frac{r}{a}\right)^{3}\left(\cos ^{3} \theta-\frac{3}{5} \cos \theta\right) \\
& \psi=2 \pi a^{2} B_{2}\left(\frac{r}{a}\right)^{3} \frac{\cos ^{3} \theta-\cos \theta}{3}+2 \pi a^{2} B_{3}\left(\frac{r}{a}\right)^{4} \frac{\cos ^{4} \theta-\frac{3}{5} \cos ^{2} \theta+\frac{1}{5}}{4} .
\end{aligned}
$$

The first null is at $r=0$ and a second null is at $r / a=c_{0} B_{2} / B_{3}$, where $c_{0}=2.357 \cdots$, and $\theta=\pi / 4$. The flux at the first null is $\psi=0$; the flux at the second null is non-zero since

$$
\frac{\cos ^{3} \theta-\cos \theta}{3}+c_{0} \frac{\cos ^{4} \theta-\frac{3}{5} \cos ^{2} \theta+\frac{1}{5}}{4}=-0.02946 \cdots
$$

when evaluated at that null. Because $\psi$ is conserved along a magnetic field line, no line that passes through the first null reaches the second. The second null is a line null and would be broken into a few point nulls if an arbitrarily small term proportional to $Y_{3}^{n}$ with $n \neq 0$ were added. But, an arbitrarily small magnetic field perturbation cannot change the trajectory of a field line of length $\sim a$ by a sufficient amount to cause field lines that passed through the first null to strike one the nulls associated with $Y_{3}$ term in the magnetic potential.

\section{C.3. Model of the solar corona}

This section will show the solar corona could be modelled in a way analogous to the simple model presented in $\S 3$. Reconnection can be driven by the motion of the surface of the Sun with a velocity $\boldsymbol{v}_{s}=\nabla \phi_{s} \times \hat{r}$. The normal, or radial, magnetic field to the surface of the Sun is denoted by $B_{a}(\theta, \varphi, t)$. Assuming the surface of the Sun to be a perfect conductor $\partial B_{a} / \partial t+\boldsymbol{v}_{s} \cdot \nabla B_{a}=0$. When the region outside the surface of the Sun is assumed to be a highly conducting and low viscosity plasma, fast magnetic reconnection will occur no matter how simple the initial normal field, even a dipole field $B_{a}(\theta, \varphi, t=0) \propto \cos \theta$, for any $\varphi$-dependent streamfunction $\phi_{s}(\theta, \varphi, t)$. 
The exterior of a sphere gives a more physical model of a corona, than the interior of a sphere. But calculating fast reconnection in the interior of a sphere is numerically simpler because the problem is bounded and every magnetic field line that enters the sphere must leave it somewhere. In an external solution, the magnetic flux leaving the sphere must equal the flux entering the sphere, but the flux need not consist of the same field lines. A major advantage of either spherical problem over a straight field model, such as that of $\S 3$, is that the magnetic field lines will be curved and hence currents that propagate along those lines will be subject to a hoop stress.

\section{Appendix D. Derivatives}

\section{D.1. Definition of $\mathrm{d} f / \mathrm{d} t$}

The total derivative with respect to time $\mathrm{d} f / \mathrm{d} t$ means moving with the plasma,

$$
\begin{aligned}
\frac{\mathrm{d} f}{\mathrm{~d} t} & \equiv\left(\frac{\partial f}{\partial t}\right)_{(x, y, z)}+\boldsymbol{v} \cdot \nabla f \\
& =\frac{\partial f}{\partial t}+\hat{z} \cdot\left(\nabla_{\perp} f \times \nabla_{\perp} \phi\right) .
\end{aligned}
$$

\section{D.2. Definition of $\mathrm{d} f / \mathrm{d} \ell$}

The derivative with respect to distance along the magnetic field lines $\mathrm{d} f / \mathrm{d} \ell$ is defined by

$$
\begin{aligned}
\frac{\mathrm{d} f}{\mathrm{~d} \ell} & \equiv \frac{\boldsymbol{B}}{B_{g}} \cdot \nabla f \\
& =\frac{\partial f}{\partial z}+\hat{z} \cdot\left(\nabla_{\perp} f \times \nabla_{\perp} H\right) .
\end{aligned}
$$

D.3. Commutator of $\nabla_{\perp}^{2}$ with $\mathrm{d} f / \mathrm{d} \ell$

$$
\begin{aligned}
\nabla_{\perp}^{2} \frac{\mathrm{d} f}{\mathrm{~d} \ell} & =\nabla_{\perp}^{2}\left(\frac{\partial f}{\partial z}+\hat{z} \cdot\left(\nabla_{\perp} f \times \nabla_{\perp} H\right)\right) \\
& =\frac{\partial \nabla_{\perp}^{2} f}{\partial z}+\hat{z} \cdot\left(\nabla_{\perp} \nabla_{\perp}^{2} f \times \nabla_{\perp} H\right)+\hat{z} \cdot\left(\nabla_{\perp} f \times \nabla \nabla_{\perp}^{2} H\right) \\
& =\frac{\mathrm{d}}{\mathrm{d} \ell} \nabla_{\perp}^{2} f+\hat{z} \cdot\left(\nabla_{\perp} K \times \nabla_{\perp} f\right) .
\end{aligned}
$$

D.4. Commutator of $\nabla_{\perp}^{2}$ with $\mathrm{d} f / \mathrm{d} t$

$$
\begin{aligned}
\nabla_{\perp}^{2} \frac{\mathrm{d} f}{\mathrm{~d} t} & =\nabla_{\perp}^{2}\left(\frac{\partial f}{\partial t}+\hat{z} \cdot\left(\nabla_{\perp} f \times \nabla_{\perp} \phi\right)\right) \\
& =\frac{\partial \nabla_{\perp}^{2} f}{\partial z}+\hat{z} \cdot\left(\nabla_{\perp} \nabla_{\perp}^{2} f \times \nabla_{\perp} \phi\right)+\hat{z} \cdot\left(\nabla_{\perp} f \times \nabla_{\perp} \nabla_{\perp}^{2} \phi\right) \\
& =\frac{\mathrm{d}}{\mathrm{d} t} \nabla_{\perp}^{2} f+\hat{z} \cdot\left(\nabla_{\perp} \Omega \times \nabla_{\perp} f\right) .
\end{aligned}
$$




$$
\begin{aligned}
\frac{\partial}{\partial t} \frac{\mathrm{d} f}{\mathrm{~d} \ell} & =\frac{\partial}{\partial z} \frac{\partial f}{\partial t}+\hat{z} \cdot\left(\nabla_{\perp} \frac{\partial f}{\partial t} \times \nabla_{\perp} H\right)+\hat{z} \cdot\left(\nabla_{\perp} f \times \nabla_{\perp} \frac{\partial H}{\partial t}\right) \\
& =\frac{\mathrm{d}}{\mathrm{d} \ell} \frac{\partial f}{\partial t}+\hat{z} \cdot\left(\nabla_{\perp} f \times \nabla_{\perp} \frac{\partial H}{\partial t}\right) .
\end{aligned}
$$

D.6. Commutator of $\mathrm{d} / \mathrm{d} \ell$ and $\mathrm{d} / \mathrm{d} t$

This derivation uses

$$
\frac{\mathrm{d} \hat{z} \cdot\left(\nabla_{\perp} f \times \nabla_{\perp} g\right)}{\mathrm{d} \ell}=\hat{z} \cdot\left(\nabla_{\perp} \frac{\mathrm{d} f}{\mathrm{~d} \ell} \times \nabla_{\perp} g\right)+\hat{z} \cdot\left(\nabla_{\perp} f \times \nabla_{\perp} \frac{\mathrm{d} g}{\mathrm{~d} \ell}\right)
$$

which follows from the coordinate invariance of $\hat{z} \cdot\left(\nabla_{\perp} f \times \nabla_{\perp} g\right)$, equation (D 10).

$$
\begin{aligned}
\frac{\mathrm{d}}{\mathrm{d} \ell} \frac{\mathrm{d} f}{\mathrm{~d} t} & =\frac{\mathrm{d}}{\mathrm{d} \ell}\left(\frac{\partial f}{\partial t}+\hat{z} \cdot\left(\nabla_{\perp} f \times \nabla_{\perp} \phi\right)\right) \\
& =\left(\frac{\partial}{\partial t} \frac{\mathrm{d} f}{\mathrm{~d} \ell}-\hat{z} \cdot\left(\nabla_{\perp} f \times \nabla_{\perp} \frac{\partial H}{\partial t}\right)\right)+\frac{\mathrm{d}}{\mathrm{d} \ell} \hat{z} \cdot\left(\nabla_{\perp} f \times \nabla_{\perp} \phi\right) \\
& =\frac{\mathrm{d}}{\mathrm{d} t} \frac{\mathrm{d} f}{\mathrm{~d} \ell}-\hat{z} \cdot\left(\nabla_{\perp} f \times \nabla_{\perp}\left(\frac{\partial H}{\partial t}-\frac{\mathrm{d} \phi}{\mathrm{d} \ell}\right)\right) \\
& =\frac{\mathrm{d}}{\mathrm{d} t} \frac{\mathrm{d} f}{\mathrm{~d} \ell}+\hat{z} \cdot\left(\nabla_{\perp} f \times \nabla_{\perp} \mathcal{N}_{B}\right) .
\end{aligned}
$$

D.7. Form of $\hat{z} \cdot\left(\nabla_{\perp} f \times \nabla_{\perp} g\right)$

$$
\hat{z} \cdot\left(\nabla_{\perp} f \times \nabla_{\perp} g\right)=\frac{\partial}{\partial x}\left(f \frac{\partial g}{\partial y}\right)-\frac{\partial}{\partial y}\left(f \frac{\partial g}{\partial x}\right) .
$$

D.8. Coordinate invariance of $\hat{z} \cdot\left(\nabla_{\perp} f \times \nabla_{\perp} g\right)$

In arbitrary coordinates $x_{a}(x, y)$ and $y_{a}(x, y)$, the gradient $\nabla_{\perp} f=\left(\partial f / \partial x_{a}\right) \nabla x_{a}+$ $\left(\partial f / \partial y_{a}\right) \nabla y_{a}$, which implies

$$
\begin{aligned}
\hat{z} \cdot\left(\nabla_{\perp} f \times \nabla_{\perp} g\right) & =\frac{\partial f}{\partial x} \frac{\partial g}{\partial y}-\frac{\partial f}{\partial y} \frac{\partial g}{\partial x} \\
& =\left(\frac{\partial f}{\partial x_{a}} \frac{\partial g}{\partial y_{a}}-\frac{\partial f}{\partial y_{a}} \frac{\partial g}{\partial x_{a}}\right) \mathcal{J}_{a} \\
\mathcal{J}_{a} & \equiv \hat{z} \cdot\left(\nabla x_{a} \times \nabla y_{a}\right) .
\end{aligned}
$$

The Jacobian of the $\left(x_{a}, y_{b}\right)$ coordinates $\mathcal{J}_{a}$ is unity when the coordinates are the starting points $\left(x_{s}, y_{s}\right)$ of magnetic field line trajectories. 


\section{REFERENCES}

Aref, H., Blake, J. R., Budisić, M., Cardoso, S. S. S., Cartwright, J. H. E., Clercx, H. J. H., El Omari, K., Feudel, U., Golestanian, R., Gouillart, E. et al. 2017 Frontiers of chaotic advection. Rev. Mod. Phys. 89, 025007.

VAn Ballegooijen, A. A. 1985 Electric currents in the solar corona and the existence of magnetostatic equilibrium. Astrophys. J. 298, 421.

Boozer, A. H. 1983 Evaluation of the structure of ergodic fields. Phys. Fluids 26, 1288-1291.

Boozer, A. H. 1999 Implications of magnetic helicity conservation. In Magnetic Helicity in Space and Laboratory Plasmas (ed. M. R. Brown, R. C. Canfield \& A. A. Pevtsov), p. 11. American Geophysical Union.

Boozer, A. H. 2004 Physics of magnetically confined plasmas. Rev. Mod. Phys. 76, 1071.

Boozer, A. H. 2010 Mathematics and Maxwell's equations. Plasma Phys. Control. Fusion 52, 124002.

Boozer, A. H. 2012 Separation of magnetic field lines. Phys. Plasmas 19, 112901.

Boozer, A. H. 2014 Formation of current sheets in magnetic reconnection. Phys. Plasmas 21, 072907.

Boozer, A. H. 2015 Non-axisymmetric magnetic fields and toroidal plasma confinement. Nucl. Fusion 55, 025001.

Boozer, A. H. 2017 Runaway electrons and ITER. Nucl. Fusion 57, 056018.

Boozer, A. H. \& Pomphrey, N. 2010 Current density and plasma displacement near perturbed rational surfaces. Phys. Plasmas 17, 110707.

Cattaneo, F., Hughes, D. W. \& Kim, E.-J. 1996 Suppression of chaos in a simplified nonlinear dynamo model. Phys. Rev. Lett. 76, 2057.

Comisso, L. \& Bhattacharjee, A. 2016 On the value of the reconnection rate. J. Plasma Phys. 82, 595820601.

Daughton, W., Nakamura, T. K. M., Karimabadi, H., Roytershteyn, V. \& Loring, B. 2014 Computing the reconnection rate in turbulent kinetic layers by using electron mixing to identify topology. Phys. Plasmas 21, 052307.

EYINK, G. L. 2015 Turbulent general magnetic reconnection. Astrophys. J 807, 137.

Eyink, G. L., LAZARIAN, A. \& VishniaC, E. T. 2011 Fast magnetic reconnection and spontaneous stochasticity. Astrophys. J. 743, 51.

Falkovich, G., GawdȩZKi, K. \& Vergassola, M. 2001 Particles and fields in fluid turbulence. Rev. Mod. Phys. 73, 913.

Greene, J. M. 1993 Reconnection of vorticity lines and magnetic lines. Phys. Fluids B 5, 2355.

HARRIS, E. G. 1962 On a plasma sheath separating regions of oppositely directed magnetic fields. Nuovo Cimento 23, 115-121.

Huang, Y.-M., Bhattacharjee, A. \& Boozer, A. H. 2014 Rapid change in field line connectivity and reconnection in stochastic magnetic fields. Astrophys. J. 793, 106.

JACKSON, J. D. 1998 Classical Electrodymanics, 3rd edn. John Wiley and Sons.

Kadomtsev, B. B. \& Pogutse, O. P. 1974 Nonlinear helical perturbations of a plasma in the tokamak. Sov. Phys. JETP 38, 283.

Lighthill, J. 1986 The recently recognized failure of predictability in Newtonian dynamics. Proc. R. Soc. Lond. Ser. A 407, 35.

Loureiro, N. F. \& UzDensky, D. A. 2016 Magnetic reconnection: from the Sweet-Parker model to stochastic plasmoid chains. Plasma Phys. Control. Fusion 58, 014021.

Newcomb, W. A. 1958 Motion of magnetic lines of force. Ann. Phys. 3, 347.

NG, C. S., Lin, L. \& BhattacharJeE, A. 2012 High-Lundquist number scaling in three-dimensional simulations of Parker's model of coronal heating. Astrophys. J. 747, 109.

Rechester, A. B. \& Rosenbluth, M. N. 1978 Electron heat-transport in a tokamak with destroyed magnetic surfaces. Phys. Rev. Lett. 40, 38.

Similon, P. L. \& SUdAN, R. N. 1989 Energy-dissipation of Alfvén-wave packets deformed by irregular magnetic-fields in solar-coronal arches. Astrophys. J. 336, 442-453.

Stern, D. P. 1966 The motion of magnetic field lines. Space Sci. Rev. 6, 147. 
Strauss, H. R. 1976 Nonlinear, 3-dimensional magnetohydrodnamics of noncircular tokamaks. Phys. Fluids 19, 134.

Woltjer, L. 1958 A theorem on force-free magnetic fields. Proc. Natl Acad. Sci. 44, 489.

Zimbardo, G., Veltri, P., Basile, G. \& Principato, S. 1995 Anomalous diffusion and Lévy random walk of magnetic field lines in three dimensional turbulence. Phys. Plasmas 2, 2653.

Zweibel, E. G. 1998 Fast reconnection of weak magnetic fields. Phys. Plasmas 5, 247.

Zweibel, E. G. \& Yamada, M. 2016 Perspectives on magnetic reconnection. Proc. R. Soc. Lond. A 472, 20160479. 